\title{
Comparison of Turbulent Thermal Diffusivity and Scalar Variance Models
}

\author{
Dennis A. Yoder* \\ NASA Glenn Research Center, Cleveland, OH, 44135, USA
}

\begin{abstract}
In this study, several variable turbulent Prandtl number formulations are examined for boundary layers, pipe flow, and axisymmetric jets. The model formulations include simple algebraic relations between the thermal diffusivity and turbulent viscosity as well as more complex models that solve transport equations for the thermal variance and its dissipation rate. Results are compared with available data for wall heat transfer and profile measurements of mean temperature, the root-mean-square (RMS) fluctuating temperature, turbulent heat flux and turbulent Prandtl number. For wall-bounded problems, the algebraic models are found to best predict the rise in turbulent Prandtl number near the wall as well as the log-layer temperature profile, while the thermal variance models provide a good representation of the RMS temperature fluctuations. In jet flows, the algebraic models provide no benefit over a constant turbulent Prandtl number approach. Application of the thermal variance models finds that some significantly overpredict the temperature variance in the plume and most underpredict the thermal growth rate of the jet. The models yield very similar fluctuating temperature intensities in jets from straight pipes and smooth contraction nozzles, in contrast to data that indicate the latter should have noticeably higher values. For the particular low subsonic heated jet cases examined, changes in the turbulent Prandtl number had no effect on the centerline velocity decay.
\end{abstract}

\section{Nomenclature}

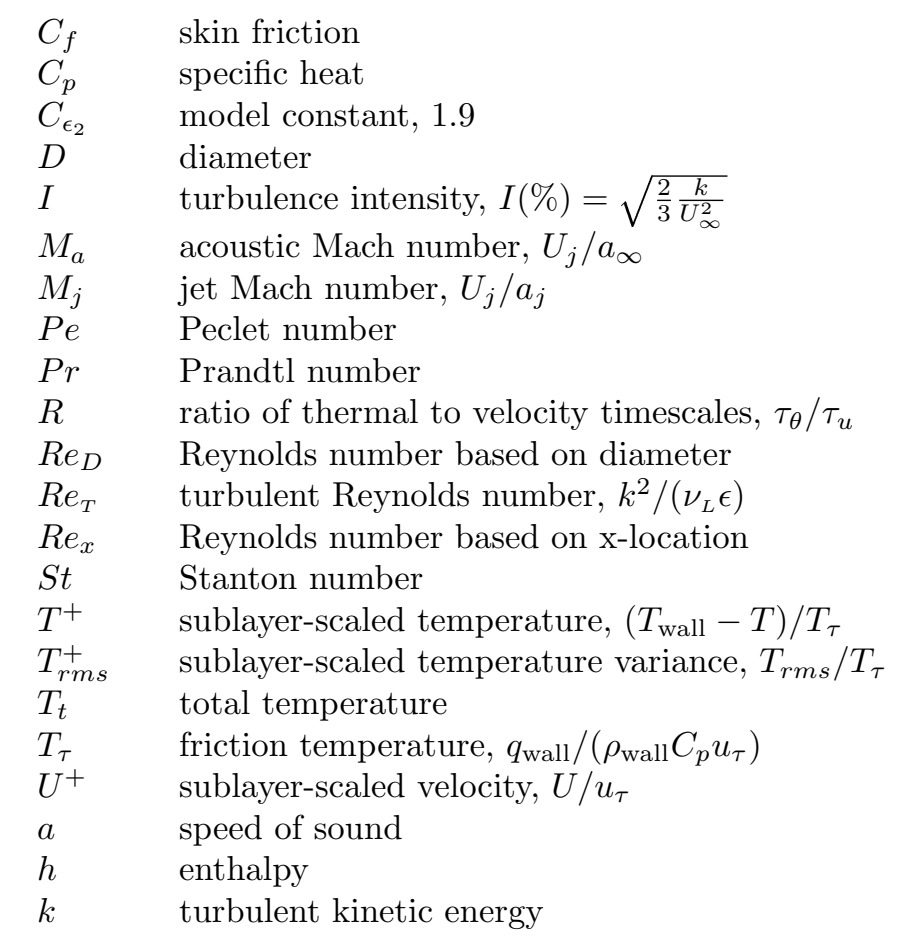

*Aerospace Engineer, Inlets and Nozzles Branch, 21000 Brookpark Road, and AIAA Associate Fellow. 


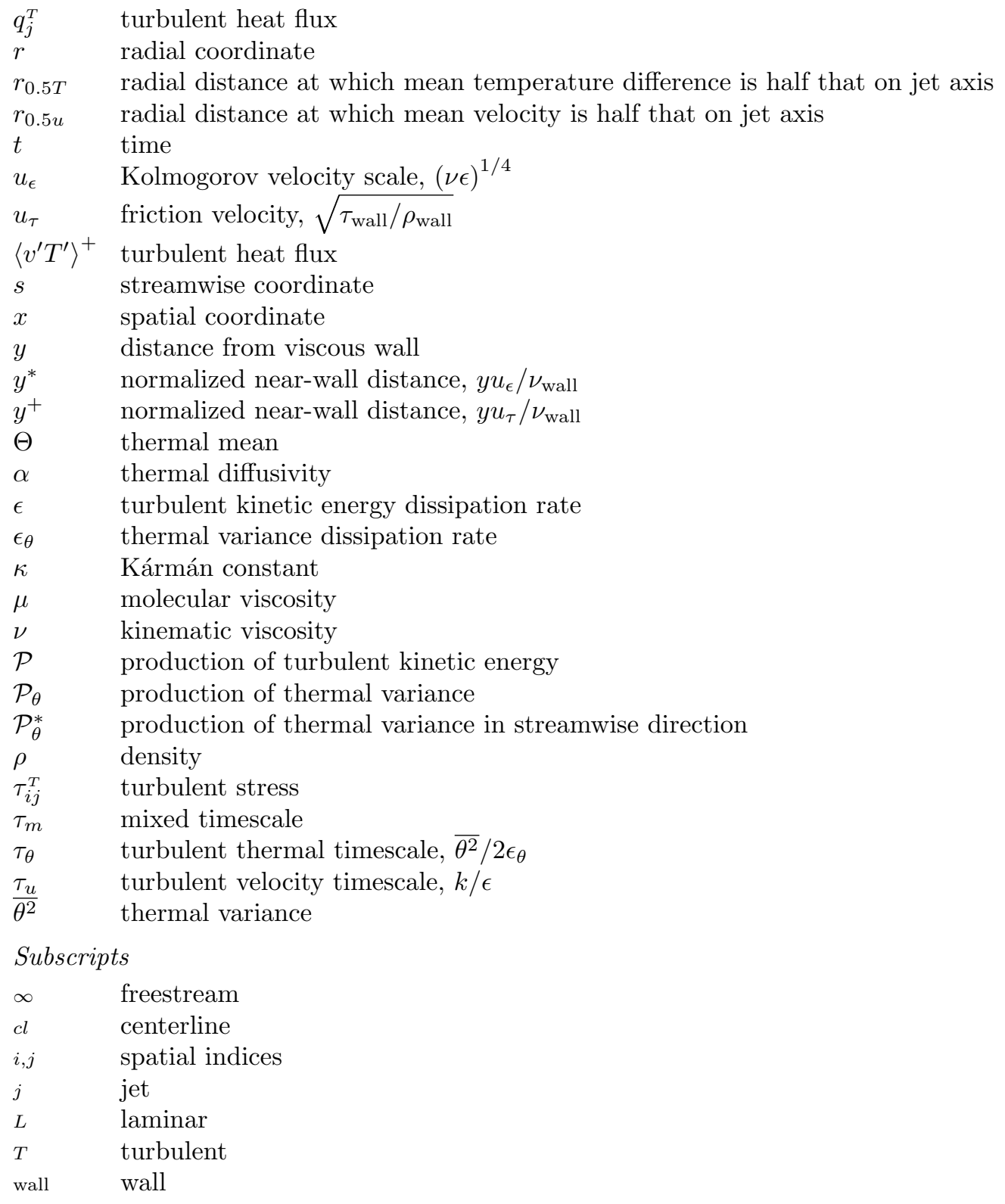

\section{Introduction}

$\mathrm{T}^{\mathrm{N}}$ simulations involving the Reynolds-averaged Navier-Stokes (RANS) equations, the turbulent heat flux is often modeled using Reynolds' analogy between momentum and energy together with an assumed constant turbulent Prandtl number, $\operatorname{Pr}_{T}$. A value of 0.9 is common for boundary layers, while a value as low as 0.5 is more appropriate in free shear flows. ${ }^{1}$ In many applications this approach works well enough for engineering accuracy, but in others the results can be sensitive to the value chosen. For example, Dembowski and Georgiadis $^{2}$ investigated the effect of adjusting the turbulent Prandtl number value on simulations of a Mach 2 heated round jet and found that while higher values of $P r_{T}$ have little effect on the velocity field, they do reduce the thermal decay as one might expect. Turbulent Prandtl number variations also affect the mixing and combustion that occurs in reacting mixing layers. Simulation results ${ }^{3}$ for a supersonic reacting mixing layer indicate that the ignition point moves upstream with increasing $\operatorname{Pr}_{T}$. Thus there exists some uncertainty in knowing what constant value to choose for a particular problem, and the optimal value for one case might not be optimal for others. In general the value of $\operatorname{Pr}_{T}$ will vary throughout the flowfield.

Kays $^{4,5}$ provides an excellent review of the fundamental theory involved in the definition of the turbulent 
Prandtl number and trends that can be observed from experimental data. Among the important things to note is that, for zero-pressure gradient boundary layers, a temperature law of the wall profile can be obtained, the slope of which is directly related to the turbulent Prandtl number. Unfortunately this profile is not universally applicable to other flows, as even the addition of favorable/adverse pressure gradients causes the slope of the temperature profile to increase/decrease respectively. Based upon available temperature data, the nominal value of turbulent Prandtl number in the log-layer region is 0.85 and is roughly constant if not slightly decreasing with distance from the wall. In the outer portion of the boundary layer, $P r_{T}$ tends towards values between $0.5-0.7$. In the near-wall region, $y^{+}<30$, the data indicate a significant increase in $\operatorname{Pr}_{T}$ to values as high as 2.0. For fluids such as air that have $\operatorname{Pr}<1.0$, precise values of turbulent Prandtl number deep in the sublayer may seem irrelevant since the molecular heat transfer becomes dominant there. However, Kays notes that while prescribing the proper value of $P r_{T}$ in the log-layer will result in the correct slope of the temperature profile, the only way to achieve the proper offset of the curve is by including higher values of $\mathrm{Pr}_{T}$ in the region $y^{+}<30$.

As with turbulence modeling, a number of different approaches have been taken in the area of turbulent heat flux modeling. Simple variable turbulent Prandtl number models such as those of References 5 and 6 are algebraic models that relate $\operatorname{Pr}_{T}$ to other local flow quantities. Other models circumvent the need for a turbulent Prandtl number by relating the turbulent thermal diffusivity to the scalar variance of a thermal quantity such as temperature, and then solving modeled transport equations for that variance and its dissipation rate. Such models are akin to $k-\epsilon$ eddy viscosity turbulence models. More sophisticated models solve transport equations for the heat fluxes themselves, much like what is done in Reynolds-stress transport models. For those cases that are amenable to Direct Numerical Simulation, no modeling is needed since the turbulent statistics (i.e., for the turbulent heat flux) can be obtained directly.

A major hindrance to the development of advanced heat transfer models has been a lack of detailed, high-quality experimental data to calibrate and validate the models. Much of this can be attributed to the difficulty in obtaining simultaneous velocity and temperature measurements to evaluate the necessary turbulent statistics. For reacting flows, development of these models is further complicated by the consideration of turbulent-chemistry interaction effects, which are sometimes included through probability density function (PDF) formulations, and the issues involved in obtaining measurements in such a harsh and dynamic environment. Direct Numerical Simulation (DNS) data is available for some cases, though typically at low Reynolds number and for configurations with some degree of pseudo-periodicity such as channel flows.

There also seems to be a lack of comprehensive model comparisons. In the realm of turbulence modeling, one can point to the works of References $7-12$ for direct and detailed analysis of various turbulence model formulations. Heat transfer models, however, are less frequently compared with other existing models, and finding independent model results for the same flow problem can be challenging. All of this makes it very difficult for one to draw any conclusions regarding which models work best across a range of cases. The objective of this paper is to provide such a comparison for one class of heat flux models, those involving the thermal diffusivity, and evaluate a number of these available models across a common set of validation cases.

\section{Review of Thermal Diffusivity Models}

Nagano and $\mathrm{Kim}^{13}$ were among the first to develop a practical eddy diffusivity heat flux model based upon transport equations for the temperature variance $\overline{t^{2}}$ and its associated dissipation rate, $\epsilon_{t}$. The formulation is similar to two-equation $k-\epsilon$ turbulence models, with terms for convection, diffusion, production, dissipation, and near-wall contributions. Production of the temperature variance is driven by gradients of the mean temperature. The dissipation rate equation, however, includes contributions from both the thermal and kinetic fields. Likewise, the thermal diffusivity is related to both the turbulent velocity and thermal time scales through a geometric average, $\tau_{m}=\sqrt{\tau_{u} \tau_{\theta}}=\tau_{u} \sqrt{2 R}$. One shortcoming of the model is that it was formulated for the case of uniform wall temperature, and does not properly reproduce the near-wall limiting behavior for configurations that have a varying wall temperature, such as those with a prescribed heat flux.

From here, model improvements follow along two primary development paths. The first path begins with the work of Nagano, Tagawa, and Tsuji ${ }^{14}$ who chose a different mixed timescale, $\tau_{m}=\tau_{u}(2 R)^{2}$, added damping functions, and recalibrated the model to improve predictions in the near-wall region including cases with non-uniform wall temperature. Youssef, Nagano, and Tagawa ${ }^{15}$ further extended this model, including a more stable numerical boundary condition for the dissipation rate. Abe, Kondoh and Nagano ${ }^{16}$ made yet another choice for the mixed timescale, using the harmonic average $\tau_{m}=2 /\left(1 / \tau_{u}+0.5 / \tau_{\theta}\right)=\tau_{u} 2 R /(R+0.5)$ 
instead. This choice implies that the shorter of the two timescales is more important for heat transfer. They also modified the model to use $y^{*}$ rather than $y^{+}$in the near-wall damping functions in order to improve results for complex flows with separation and reattachment. Nagano, Hattori and Abe ${ }^{17}$ improved the numerical stability of Abe's model by reformulating it to apply $\epsilon_{t}=0$ at the wall. Deng, Wu and Xi ${ }^{18}$ took a different approach in modeling several terms, including the production term of the dissipation rate equation, and modified the near-wall damping terms for flows with constant temperature or heat flux. However, the timescale is again based on the geometric average and many of the model coefficients remain the same as those used by Youssef.

The different relations described above for the mixed timescale are plotted in Figure 1. One should note that the timescale ratio $R$ in an equilibrium boundary layer is relatively constant, with a nominal value of 0.5 . In that situation, all of the proposals for the mixed timescale yield very similar values and produce similar results. Such findings were demonstrated by Abe, Kondoh and Nagano. ${ }^{16}$ Away from this equilibrium state the timescale relations differ, with the $\tau_{m}=\tau_{u}(2 R)^{2}$ model of Nagano, Tagawa, and Tsuji providing the greatest variation.

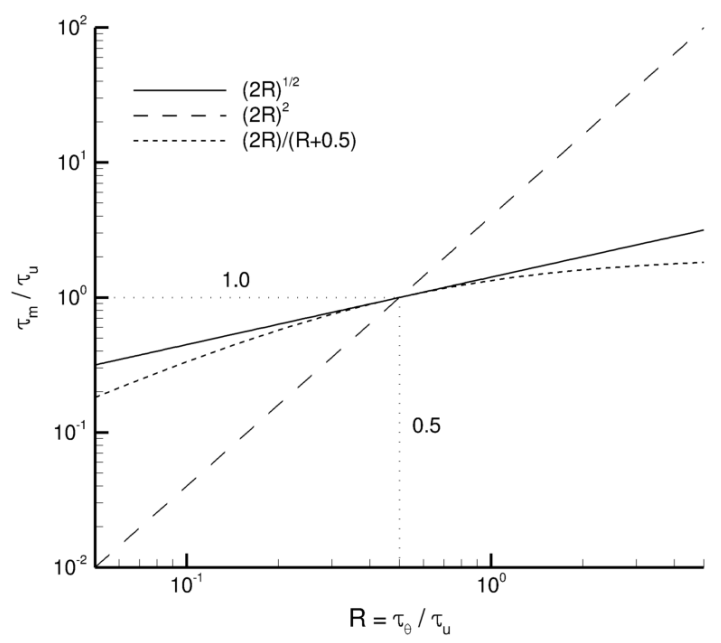

Figure 1: Mixed timescale relations.

The second development path begins with the work of Sommer, So, and Lai ${ }^{19}$ to add proper incompressible near-wall terms to the Nagano-Kim ${ }^{13}$ model. Sommer, So and Zhang ${ }^{20}$ modified these terms to account for compressible flow. Chidambaram, Dash and Kenzakowski ${ }^{21}$ extended the model for application to jets by modifying some of the model coefficients. Kenzakowski ${ }^{22}$ explored the use of a modified timescale in the near-field region of the jet in order to increase the thermal mixing and shorten the thermal potential core length. A series of additional papers by this same group eventually led to the model of Brinckman, Calhoon, and Dash. ${ }^{23}$ This model has been modified to use $y^{*}$ rather than $y^{+}$in the near-wall damping functions, and solves for the scalar variance of internal energy rather than temperature for multi-species flow. So and Sommer ${ }^{24}$ extended the model of Sommer-So-Lai ${ }^{19}$ to sensitize the model to molecular Prandtl numbers that are not close to one. Sommer, So and Zhang ${ }^{25}$ later extended this model to supersonic flow. In another work, Sommer, So and Zhang ${ }^{26}$ evaluated the assumption that $\overline{t^{2}}=0$ at the wall and found that specifying a non-zero boundary value has essentially no effect on the mean temperature and integral heat transfer properties. The effect on the turbulence statistics were limited to a very small region near the wall. They therefore recommend the continued use of $\overline{t^{2}}=0$, since it is much simpler to apply.

All of the models described above are derived from the low speed energy equation where the pressure gradient and energy dissipation terms ${ }^{\text {a }}$ are neglected. Accounting for these additional contributions, Xiao ${ }^{29}$ re-derived the exact equations for the enthalpy variance and dissipation rate and developed a model similar in form and compatible with the $k-\zeta$ turbulence model. ${ }^{30}$ That work demonstrated a significant improvement in heat transfer predictions for shock wave/boundary layer interaction problems. In Reference 31, the model was extended to account for chemistry source terms and applied it to a scramjet combustion simulation.

${ }^{a}$ See, for example, Cebeci and Bradshaw ${ }^{27}$ or Anderson. ${ }^{28}$ 


\section{Model Descriptions}

Within the context of the RANS equations, ${ }^{1}$ the turbulent heat flux vector is defined as

$$
q_{j}^{T} \equiv \overline{\rho u_{j}^{\prime \prime} h^{\prime \prime}}
$$

and must be modeled. This is typically done by relating the heat flux to a thermal diffusivity and the mean thermal gradient, much like the Boussinesq approximation relates the Reynolds' stress to the eddy viscosity and the mean velocity gradient.

$$
q_{j}^{T}=-\bar{\rho} \alpha_{T} \frac{\partial \widetilde{h}}{\partial x_{j}}
$$

Models for the heat transfer must then provide an expression for the thermal diffusivity. As with turbulence models, these may be classified in terms of the number of additional transport equations that must be solved.

\section{III.A. Zero-Equation Models}

Many zero-equation (i.e., algebraic) models relate the eddy diffusivity to the turbulent viscosity and a turbulent Prandtl number.

$$
\bar{\rho} \alpha_{T}=\frac{\mu_{T}}{P r_{T}}
$$

The simplest expression for the turbulent Prandtl number is to prescribe a constant value. As mentioned in the introduction, a value of 0.9 is common for boundary layers, while a value as low as 0.5 is more appropriate in free shear flows. In applying a constant value of $P r_{T}$, an analogy is assumed between the turbulent heat and momentum transfer.

Wassel and Catton ${ }^{6}$ developed the following model for the turbulent Prandtl number

$$
\operatorname{Pr}_{T}=\frac{C_{3}}{C_{1} \operatorname{Pr}}\left[1-\exp \left(\frac{-C_{4}}{\left(\mu_{T} / \mu_{L}\right)}\right)\right]\left[1-\exp \left(\frac{-C_{2}}{\left(\mu_{T} / \mu_{L}\right) \operatorname{Pr}}\right)\right]^{-1}
$$

where the coefficients are given as $C_{1}=0.21, C_{2}=5.25, C_{3}=0.20$, and $C_{4}=5.00$.

The model of Kays and Crawford ${ }^{5}$ is an algebraic formulation that relates the turbulent Prandtl number to a turbulent Peclet number, $P e_{T}=\left(\mu_{T} / \mu_{L}\right) \operatorname{Pr}$.

$$
P r_{T}=\left\{\frac{1}{2 P r_{T_{\infty}}}+\frac{C P e_{T}}{\sqrt{P r_{T_{\infty}}}}-\left(C P e_{T}\right)^{2}\left[1-\exp \left(\frac{-1}{C P e_{T} \sqrt{P r_{T_{\infty}}}}\right)\right]\right\}^{-1}
$$

In the relation above, $P r_{T_{\infty}}$ is the value of the turbulent Prandtl number far from the wall. Based upon experimental data, the model constants are set as $C=0.3$ and $P r_{T_{\infty}}=0.85$.

In the discussion that follows, these models shall be referred to by their initials: WC and KC.

\section{III.B. Two-Equation Models}

Two-equation models solve transport equations for some measure of the thermal variance $\left(\overline{\theta^{2}}\right)$ and its dissipation rate $\left(\epsilon_{\theta}\right)$. Usually the thermal quantity is temperature, although enthalpy, internal energy, and even stagnation enthalpy have been successfully applied by making the corresponding change in the mean thermal quantity $(\Theta)$ used in the production terms below.

$$
\begin{aligned}
& \frac{\partial\left(\bar{\rho} \overline{\theta^{2}}\right)}{\partial t}+\frac{\partial\left(\bar{\rho} \widetilde{u}_{j} \overline{\theta^{2}}\right)}{\partial x_{j}}=\frac{\partial}{\partial x_{j}}\left[\bar{\rho}\left(\alpha_{L}+\frac{\alpha_{T}}{\sigma_{\theta}}\right) \frac{\partial k_{t}}{\partial x_{j}}\right]+2 \mathcal{P}_{\theta}-2 \rho \epsilon_{\theta} \\
& \frac{\partial\left(\bar{\rho} \epsilon_{\theta}\right)}{\partial t}+\frac{\partial\left(\bar{\rho} \widetilde{u}_{j} \epsilon_{\theta}\right)}{\partial x_{j}}=\frac{\partial}{\partial x_{j}}\left[\bar{\rho}\left(\alpha_{L}+\frac{\alpha_{T}}{\sigma_{\epsilon_{\theta}}}\right) \frac{\partial \epsilon_{\theta}}{\partial x_{j}}\right]+\left[C_{d 1} f_{d 1} \frac{\epsilon_{\theta}}{\overline{\theta^{2}}}+C_{d 2} f_{d 2} \frac{\epsilon}{k}\right] \mathcal{P}_{\theta}+C_{d 3} f_{d 3} \frac{\epsilon}{k}\left(\frac{\mathcal{P}}{\bar{\rho} \epsilon}\right) \bar{\rho} \epsilon_{\theta} \\
& -\left[C_{d 4} f_{d 4} \frac{\hat{\epsilon}_{\theta}}{\overline{\theta^{2}}}+C_{d 5} f_{d 5} \frac{\hat{\epsilon}}{k}\right] \bar{\rho} \epsilon_{\theta}+L_{\epsilon_{\theta}}
\end{aligned}
$$




$$
\begin{array}{rlrl}
L_{\epsilon_{\theta}} & =f_{\epsilon_{\theta}} \bar{\rho}\left[\left(C_{d 4}-4\right) \frac{\hat{\epsilon}_{\theta}}{\overline{\theta^{2}}} \epsilon_{\theta}+C_{d 5} \frac{\hat{\epsilon}}{k} \epsilon_{\theta}-\frac{\left(\epsilon_{\theta}^{*}\right)^{2}}{\overline{\theta^{2}}}+\left(2-C_{d 1}-C_{d 2} \operatorname{Pr}\right) \frac{\epsilon_{\theta}}{\overline{\theta^{2}}} \frac{\mathcal{P}_{k_{\theta}}^{*}}{\bar{\rho}}\right] \\
\mathcal{P} & =\tau_{i j}^{T} \frac{\partial \widetilde{u}_{i}}{\partial x_{j}} \approx \mu_{T} \frac{\partial \widetilde{u}_{i}}{\partial x_{j}} \frac{\partial \widetilde{u}_{i}}{\partial x_{j}} & \hat{\epsilon}=\epsilon-2 \nu_{L}\left(\frac{\partial k}{\partial y}\right)^{2} f_{\hat{\epsilon}} \\
\mathcal{P}_{\theta}=\bar{\rho} \alpha_{T} \frac{\partial \widetilde{\Theta}}{\partial x_{j}} \frac{\partial \widetilde{\Theta}}{\partial x_{j}} & \hat{\epsilon}_{\theta}=\epsilon_{\theta}-\alpha_{L}\left(\frac{\partial \overline{\theta^{2}}}{\partial y}\right)^{2} f_{\hat{\epsilon}_{\theta}} \\
\mathcal{P}_{\theta}^{*}=\bar{\rho} \alpha_{T} \frac{\partial \widetilde{\Theta}}{\partial x} \frac{\partial \widetilde{\Theta}}{\partial x} & \hat{\epsilon}_{\theta}^{*}=\epsilon_{\theta}-\frac{\alpha_{L} \frac{\overline{\theta^{2}}}{y^{2}} f_{\hat{\epsilon}_{\theta}^{*}}}{}
\end{array}
$$

Wall boundary conditions are given by:

$$
\overline{\theta^{2}}=0 \quad \epsilon_{\theta}=\alpha_{L}\left(\frac{\partial \sqrt{\overline{\theta^{2}}}}{\partial y}\right)^{2}
$$

The thermal diffusivity is then computed from a mixed timescale, which is a combination of the turbulent velocity and thermal timescales.

$$
\alpha_{T}=C_{\lambda} f_{\lambda} k \tau_{m}
$$

Table 1: Closure coefficients.

\begin{tabular}{|lcccccccc|}
\hline Model & $C_{\lambda}$ & $C_{d 1}$ & $C_{d 2}$ & $C_{d 3}$ & $C_{d 4}$ & $C_{d 5}$ & $\sigma_{\theta}$ & $\sigma_{\epsilon_{\theta}}$ \\
\hline AKN & 0.10 & 1.90 & 0.0 & 0.60 & 2.0 & 0.9 & 1.60 & 1.60 \\
DWX & 0.10 & 2.34 & 0.0 & 0.00 & 2.0 & 0.9 & 1.00 & 1.00 \\
SSZ & 0.10 & 2.00 & 0.0 & 0.72 & 2.2 & 0.8 & 0.75 & 1.45 \\
BCD & 0.14 & 2.00 & 0.0 & 0.72 & 2.2 & 0.8 & 1.00 & 1.00 \\
\hline
\end{tabular}

In this work, four transport models have been selected for comparison, based on their unique similarities and differences. These models are as follows:

- AKN - Abe, Kondoh, and Nagano ${ }^{16}$

- DWX - Deng, Wu, and $\mathrm{Xi}^{18}$

- SSZ - Sommer, So, and Zhang ${ }^{20}$

- BCD - Brinckman, Calhoon, and Dash ${ }^{23}$

Table 1 summarizes the model closure coefficients, while Table 2 lists the definitions for the timescale and damping functions. The AKN and DWX models are similar in that neither use the near wall modifications provided by $f_{\epsilon_{\theta}}, f_{\hat{\epsilon}}, f_{\hat{\epsilon}_{\theta}}$, or $f_{\hat{\epsilon}_{\theta}^{*}}$. They also use very similar forms for many of the other damping functions. Where they differ is in the choice of $\tau_{m}, f_{\lambda}$, and $f_{d 1}$. With $C_{d 3}=0$ in the DWX model, the contribution from the production of turbulent kinetic energy, $\mathcal{P}$, is neglected and the only source of production in Eq. (7) is from the thermal production term, $\mathcal{P}_{\theta}$. While the AKN model uses $\mathcal{P}_{\theta}$ in combination with the thermal timescale, the $f_{d 1}$ of DWX effectively uses a mixed timescale instead. The SSZ and BCD models are also very similar. Both models do use the near wall correction terms and share same damping functions. The primary difference between them is in the model coefficients and the use of $y^{+}$versus $y^{*}$ in $f_{\lambda}$. In separated flow, $y^{+} \rightarrow 0$ near the separation and reattachment locations, while $y^{*}$ is more well behaved.

\section{Numerical Method}

The results presented below have been computed with the Wind-US ${ }^{32}$ general purpose, compressible Navier-Stokes flow solver. The mean flow equations are solved on structured grids using second order 
Table 2: Timescale and damping function definitions.

\begin{tabular}{|c|c|}
\hline$A K N-A b e$, Kondoh, and Nagano ${ }^{16}$ & $D W X-$ Deng, $W u$, and $X i^{18}$ \\
\hline$\tau_{m}=\tau_{u}\left[\frac{2 R}{0.5+R}+\frac{3 \sqrt{2 R}}{\operatorname{Pr} R e_{T}^{3 / 4}} \exp \left[-\left(\operatorname{Re}_{T} / 200\right)^{2}\right]\right.$ & $\tau_{m}=\tau_{u} \sqrt{2 R}$ \\
\hline$f_{\lambda}=\left[1-\exp \left(-y^{*} / 14\right)\right]\left[1-\exp \left(-y^{*} \sqrt{\operatorname{Pr}} / 14\right)\right]$ & $f_{\lambda}=\left[1+3 R e_{T}^{-3 / 4}\right]\left[1-\exp \left(-y^{*} / 16\right)\right]^{2}$ \\
\hline$f_{d 1}=\left[1-\exp \left(-y^{*}\right)\right]^{2}$ & $f_{d 1}=(2 R)^{-1 / 2}$ \\
\hline$f_{d 2}=1$ & $f_{d 2}=1$ \\
\hline$f_{d 3}=1$ & $f_{d 3}=1$ \\
\hline$f_{d 4}=\left[1-\exp \left(-y^{*}\right)\right]$ & $f_{d 4}=\left[1-\exp \left(-y^{*} / 17\right)\right]^{2}$ \\
\hline$f_{d 5}=\frac{1}{C_{d 2}}\left[C_{\epsilon_{2}} f_{2}-1\right]\left[1-\exp \left(-y^{*} / 5.7\right)\right]^{2}$ & $f_{d 5}=\frac{1}{C_{d 2}}\left[C_{\epsilon_{2}} f_{2}-1\right]\left[1-\exp \left(-y^{*} / 5.8\right)\right]^{2}$ \\
\hline$f_{2}=1-0.3 \exp \left[-\left(R e_{T} / 6.5\right)^{2}\right]$ & $f_{2}=1-0.3 \exp \left[-\left(R e_{T} / 6.5\right)^{2}\right]$ \\
\hline$f_{\epsilon_{\theta}}=0$ & $f_{\epsilon_{\theta}}=0$ \\
\hline$f_{\hat{\epsilon}}=0$ & $f_{\hat{\epsilon}}=0$ \\
\hline$f_{\hat{\epsilon}_{\theta}}=0$ & $f_{\hat{\epsilon}_{\theta}}=0$ \\
\hline$f_{\hat{\epsilon}_{\theta}^{*}}=0$ & $f_{\hat{\epsilon}_{\theta}^{*}}=0$ \\
\hline SSZ - Sommer, So, and Zhang 20 & $B C D-$ Brinckman, Calhoon, and Dash ${ }^{23}$ \\
\hline$\tau_{m}=\tau_{u} \sqrt{2 R}$ & $\tau_{m}=\tau_{u} \sqrt{2 R}$ \\
\hline$f_{\lambda}=f_{\epsilon_{\theta}} \frac{0.1}{R e_{T}^{1 / 4}}+\left[1-\exp \left(-y^{+} / 45\right)\right]^{2}$ & $f_{\lambda}=f_{\epsilon_{\theta}} \frac{0.1}{R e_{T}^{1 / 4}}+\left[1-\exp \left(-y^{*} / 45\right)\right]^{2}$ \\
\hline$f_{\epsilon_{\theta}}=\exp \left[-\left(R e_{T} / 80\right)^{2}\right]$ & $f_{\epsilon_{\theta}}=\exp \left[-\left(R e_{T} / 80\right)^{2}\right]$ \\
\hline$f_{d 1}=1$ & $f_{d 1}=1$ \\
\hline$f_{d 2}=1$ & $f_{d 2}=1$ \\
\hline$f_{d 3}=1$ & $f_{d 3}=1$ \\
\hline$f_{d 4}=1$ & $f_{d 4}=1$ \\
\hline$f_{d 5}=1$ & $f_{d 5}=1$ \\
\hline$f_{\epsilon_{\theta}}=\exp \left[-\left(R e_{T} / 80\right)^{2}\right]$ & $f_{\epsilon_{\theta}}=\exp \left[-\left(R e_{T} / 80\right)^{2}\right]$ \\
\hline$f_{\hat{\epsilon}}=1$ & $f_{\hat{\epsilon}}=1$ \\
\hline$f_{\hat{\epsilon}_{\theta}}=1$ & $f_{\hat{\epsilon}_{\theta}}=1$ \\
\hline$f_{\hat{\epsilon}_{\theta}^{*}}=1$ & $f_{\hat{\epsilon}_{\theta}^{*}}=1$ \\
\hline
\end{tabular}


upwinding, while the $k-\epsilon$ and $\overline{\theta^{2}}-\epsilon_{\theta}$ equations are each solved in a loosely coupled fashion using the first-order upwinding scheme described in Reference 33. Information from the thermal variance models is fed back to the mean flow equations through the calculation of the turbulent Prandtl number. Sutherland's law is used to compute the molecular viscosity.

In the implementation of the $\mathrm{KC}$ model, the value of $P r_{T_{\infty}}$ is not hard-coded to 0.85 as specified in the original formulation, but instead is set to the freestream turbulent Prandtl number specified by the user. This allows for greater flexibility in applying the model.

Since each of the thermal variance models was originally developed for use with a specific $k-\epsilon$ turbulence model, those turbulence models have been added to the Wind-US flow solver. However, the use of different turbulence models complicates the present analysis, as differences in the turbulence model predictions for skin friction feed into the near-wall normalizations used in the presentation of the thermal quantities. Therefore, for the boundary layer and pipe flow problems the $k-\epsilon$ model of Abe, Kondoh, and Nagano ${ }^{34}$ is used. This is the model used by AKN and DWX and has been demonstrated to provide very accurate near-wall predictions. For jet problems, the $k-\epsilon$ model of BCD is chosen as this model provides consistent results for free shear flows.

Convergence issues were experienced with the SSZ and BCD models for wall-bounded flows and the resulting temperature variance profiles differed significantly from References 19, suggesting that there is an inconsistency in the current implementation of the near-wall source terms for these models. For this reason, those results will not be included in the current model assessment. Results for the jet cases, however, were found to be consistent with previous studies such as References 35 and are included in the current assessment.

Many of the previous validation studies have used parabolic marching codes with inflow conditions prescribed from experimental data at the first measurement station. While such procedures are good for model validation, they do not provide a practical assessment of one's ability to simulate cases where less complete information is available. In the present simulations, a uniform inflow boundary is placed well upstream of the region of interest and adjusted as necessary to match integral boundary layer properties such as momentum thickness at the downstream measurement station. These calculations therefore include the entire viscous development region, including the leading edge for boundary layer and pipe flows or the internal nozzle for jets. For the boundary layer cases examined, the experiments used a trip near the leading edge to cause the flow to be fully turbulent. In simulating these cases, it was necessary to prescribe elevated inflow turbulence values on the inflow plane. Otherwise, the $k-\epsilon$ model would undergo a "numerical transition" before becoming fully turbulent. On the far-field freestream boundary, zero gradient conditions were applied to the turbulence variables.

In general, the grids used in this study contained 200 points in the transverse direction to resolve the mean velocity and thermal shear layers. For the wall-bounded problems, a near-wall $y^{+}$spacing of 0.1 or less was used. For the jet flows, the near-wall spacing was relaxed to $y^{+}$less than 1.0. Grid sequencing was used to evaluate grid sensitivity of the models. Convergence was determined by monitoring plots of the physical quantities of interest for each case. These quantities included streamwise variations along the wall or centerline, as well as profiles at various streamwise locations. For each case, the mean flow values tended to converge first, while the downstream turbulent profiles took longer.

\section{Results}

Results are presented for three classes of problems: boundary layers, pipe flow, and jets. Solutions are evaluated based on agreement with available data for the mean velocity and temperature, thermal variance, heat flux, and turbulent Prandtl number. In some instances multiple experimental data sets are used in order to provide a more complete assessment of the models.

\section{V.A. Boundary Layers}

The flat plate boundary layer is a classic case for turbulence model benchmarking. In the low-speed experiment by Blackwell, Kays, and Moffat, ${ }^{36}$ the plate is held at a constant temperature $\left(14^{\circ} \mathrm{C}\right.$ above ambient) from the leading edge so that the momentum and thermal boundary layers develop simultaneously. The freestream velocity was $9.65 \mathrm{~m} / \mathrm{s}$. At the downstream station used for comparison, $x=17.78 \mathrm{~mm}$, the momentum thickness Reynolds number was 2481. In order to ensure the flow was fully turbulent from the leading edge, the present simulations applied a turbulence intensity of $5 \%$ and $\mu_{T} / \mu_{L}=20$ at the inflow plane. 
The skin friction along the plate is presented in Figure 2. The result for this low speed case is found to be insensitive to the choice of turbulent Prandtl model. The curve follows the expected trend, though the values appear to be about three percent high. Additional improvement might be obtained through further adjustment of the inflow turbulence level.

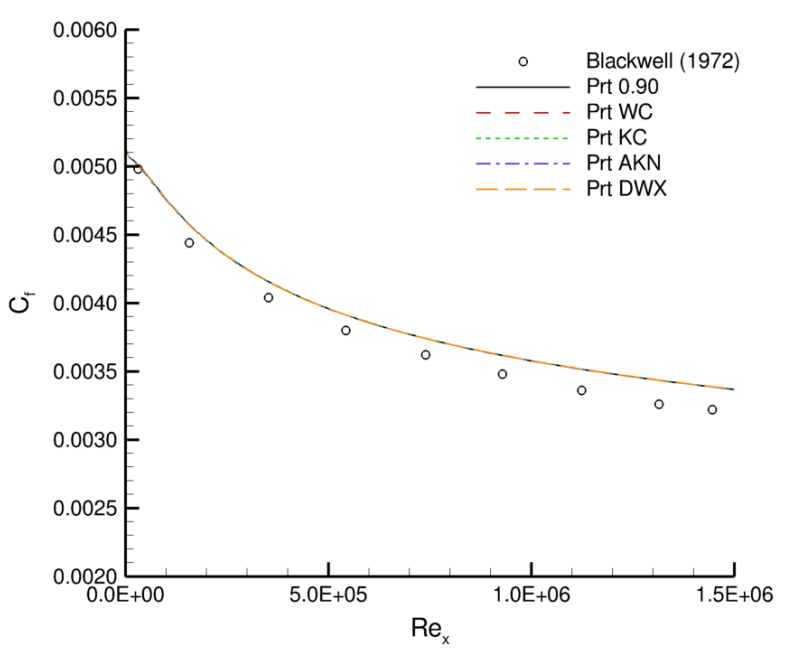

Figure 2: Skin friction for flat plate boundary layer.

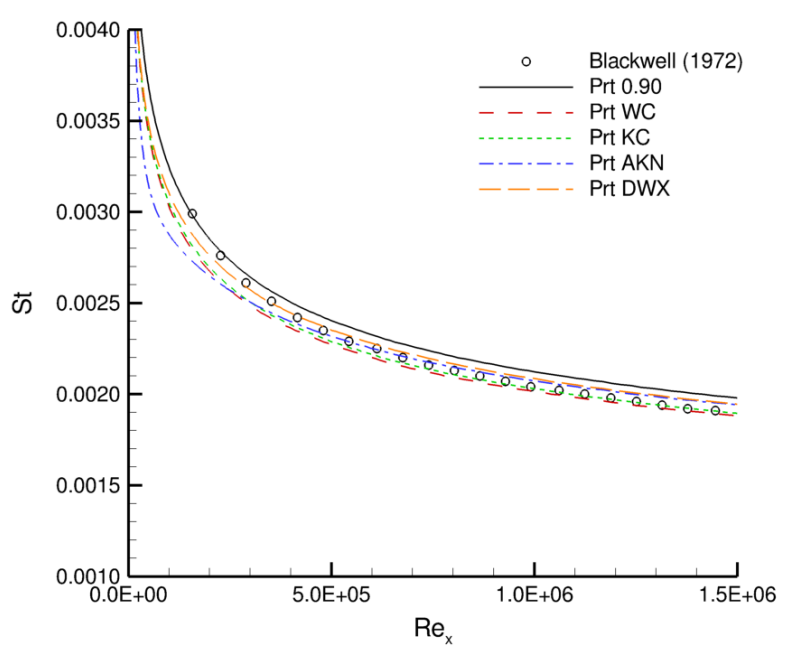

Figure 3: Stanton number for flat plate boundary layer.

The Stanton number along the plate, shown in Figure 3, indicates a spread of five percent between the various models that encapsulates the available data. $\operatorname{Pr}_{T} 0.90$ provides the highest values, and generally over-predicts the heating. The $\mathrm{WC}$ and $\mathrm{KC}$ zero-equation models are very similar and provide the lowest values. The AKN and DWX two-equation models fall somewhere in the middle, though the AKN model predicts significantly lower values very near the leading edge.

Figures 4 and 5 compare the velocity and temperature profiles plotted in inner-layer coordinates. Again the velocity field results are found to be independent of $\mathrm{Pr}_{T}$, and the profile is in excellent agreement with the experimental data. The profile also obeys the relation $u^{+}=y^{+}$in the sublayer and matches the slope of the law of the wall in the log layer. The temperature profiles all match the sublayer relation $T^{+}=\operatorname{Pr} y^{+}$. However, differences in the log-layer behavior are evident. Since the wall heat flux is used in obtaining the normalized quantity $T^{+}$, the trend in the profiles is opposite that for the Stanton number with $P r_{T}=0.90$ now providing the lowest values, $\mathrm{WC}$ and $\mathrm{KC}$ providing the highest values, and AKN and DWX falling somewhere in the middle. Of these, the WC and $\mathrm{KC}$ zero-equation models clearly provide the best agreement with the data.

As explained by Kays, ${ }^{4}$ for this type of flow the slope of the log-layer temperature profile is directly proportional to the turbulent Prandtl number.

$$
\frac{P r_{T}}{\kappa}=\frac{0.85}{0.41}=2.075
$$

Figure 6 compares the turbulent Prandtl number profiles. The experimental data show large values $\left(\operatorname{Pr}_{T} \approx 2\right)$ near the wall, with a sharp reduction in the outer part of the sublayer through $y^{+} \approx 20$. Values in the lower part of the log layer are nominally 0.9 , but then gradually decrease to a value of 0.7 near the boundary layer edge. As can be seen, use of a constant value of 0.9 is a rather crude approximation. The zero-equation models of WC and $\mathrm{KC}$ do an excellent job capturing the near wall region and the lower part of the log layer. The two-equation models of AKN and DWX accurately predict the gradual variation through the log-layer region. However, the predicted rise in turbulent Prandtl number near the wall is underpredicted and located too deep within the viscous sublayer. None of the models capture the trend of lower $\operatorname{Pr}_{T}$ in the outer part of the boundary layer, though this might be possible by reducing $P r_{T_{\infty}}$ specified with the KC model. The trends observed here are consistent with Kays assertion that higher values of $\operatorname{Pr}_{T}$ are necessary to obtain the proper offset of the log-layer temperature profile. 


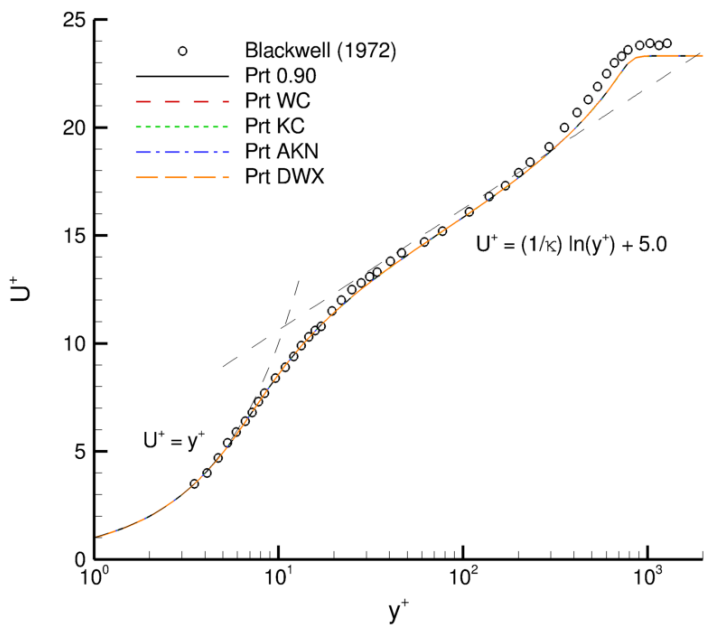

Figure 4: Velocity profile for flat plate boundary layer.

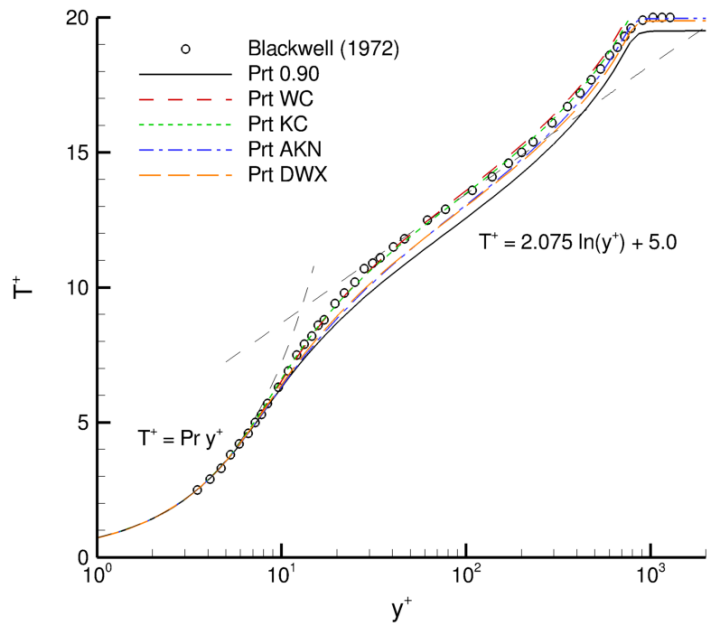

Figure 5: Temperature profile for flat plate boundary layer.

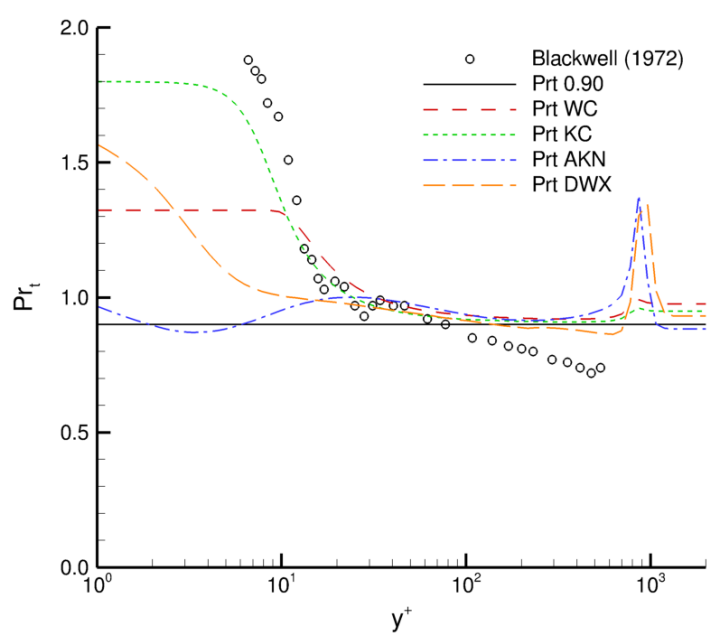

Figure 6: Turbulent Prandtl number profile for flat plate boundary layer. 
To further assess the models for this class of flow, simulations were performed for the heated boundary layer of Gibson, et al. ${ }^{37-39}$ In this experiment, depicted in Figure 7, the boundary layer develops along a length $\Delta s=1219 \mathrm{~mm}$ of the flat heated lower wall of an open circuit wind tunnel. The downstream section of the tunnel, also of length $\Delta s=1219 \mathrm{~mm}$, has a mild convex curvature with a nominal radius of $2.44 \mathrm{~m}$ that is also heated to the same constant wall temperature $\left(14^{\circ} \mathrm{C}\right.$ above ambient $)$. The upstream height of the tunnel is $102 \mathrm{~mm}$. At the point where curvature begins, $s=0 \mathrm{~mm}$, the tunnel height expands suddenly to $105 \mathrm{~mm}$ to reduce the negative pressure gradient. Flow conditions at the first measurement station, $s=-705 \mathrm{~mm}$ in the flat section, were reported as $U=22.3 \mathrm{~m} / \mathrm{s}$ and the momentum thickness Reynolds number at $s=0 \mathrm{~mm}$ was 3300 . For this case, a turbulence intensity of $4 \%$ and $\mu_{T} / \mu_{L}=10$ were applied at the inflow plane.

Results for the skin friction and heat transfer are shown in Figure 8. The skin friction is found to be in excellent agreement with the data along the flat section, but is significantly overpredicted in the downstream curved section. This is a known limitation with $k-\epsilon$ turbulence models, which are not well sensitized to streamwise curvature unless additional correction terms are added to the model. Results for the heat flux are also found to be in generally good agreement with the data. The variation among the turbulent Prandtl models is consistent with that observed for the flat plate case, with the WC and $\mathrm{KC}$ models again predicting the lowest values.

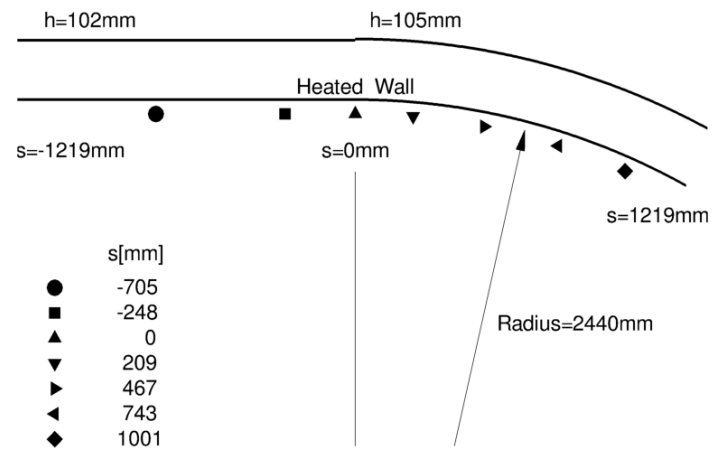

Figure 7: Schematic of Gibson boundary layer experiment.

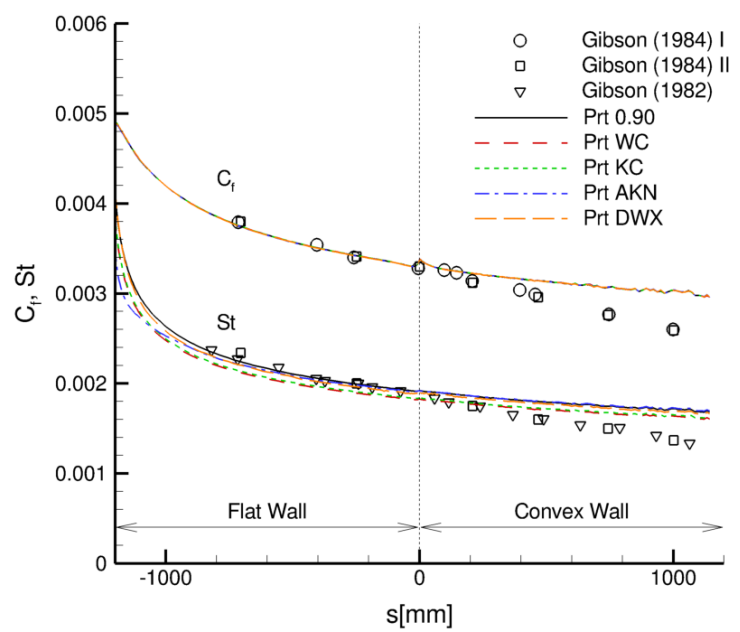

Figure 8: Skin friction and Stanton number for Gibson boundary layer.

Next, we examine the profiles within the flow. Due to the shortcomings of the $k-\epsilon$ turbulence model in the curved region, we shall limit the analysis to the last profile location ( $\mathrm{s}=-248 \mathrm{~mm}$ ) along the flat section. Figure 9 compares the velocity profile, which is again in excellent agreement with the data. The temperature profile is given in Figure 10 and the results are nearly identical to those for the flat plate case of Blackwell.

The experiment of Gibson recorded additional data that is useful for comparison. Figure 11 shows the root-mean-square (RMS) of the fluctuating temperature. Though the thermal variance models mimic the basic shape of the profile, lack of near-wall data makes it difficult to draw any conclusions. Fortunately, in another heated boundary layer experiment, Subramanian and Antonia ${ }^{40}$ collected detailed near-wall data at several streamwise locations. That data is plotted against the current results in Figure 12. Their data not only agrees well with that of Gibson, it also collapses very nicely in the near-wall region with a peak $T_{r m s}^{+}$ value of 2.0 near $y^{+} \approx 16$. The model results capture both of these features as well as the sharp decline near the edge of the boundary layer. However, the models do over-predict the values in a large portion of the outer boundary layer.

The turbulent heat flux profiles are shown in Figure 13. The data of Gibson ${ }^{38}$ is again limited to the outer portion of the boundary layer. Unfortunately Subramanian and Antonia ${ }^{40}$ did not measure the turbulent heat flux and so their data cannot be used to evaluate the near-wall behavior. Compared with the data shown, the models tend to overpredict the turbulent heat flux. Turbulent Prandtl number profiles are plotted in Figure 14. The data is again fairly limited, with values that are higher than those reported by Blackwell. ${ }^{36}$ The model predictions are very consistent with those for the previous flat plate case. 


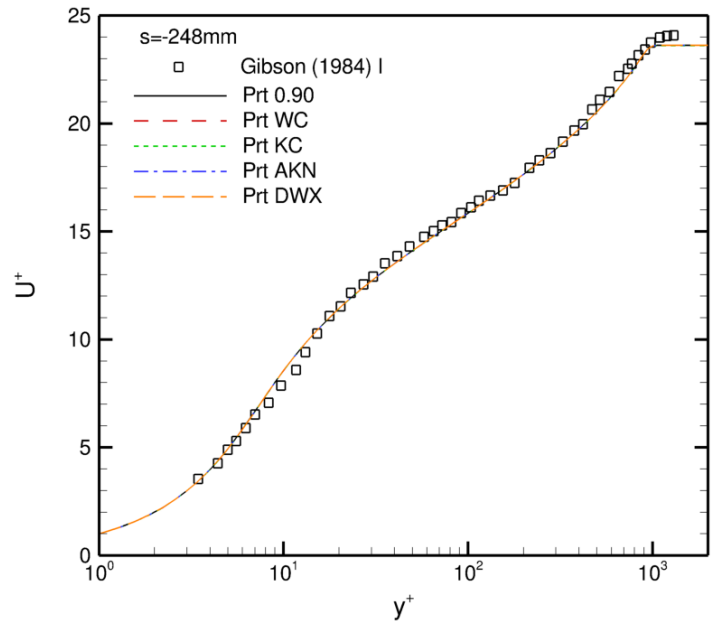

Figure 9: Velocity profile for Gibson boundary layer.

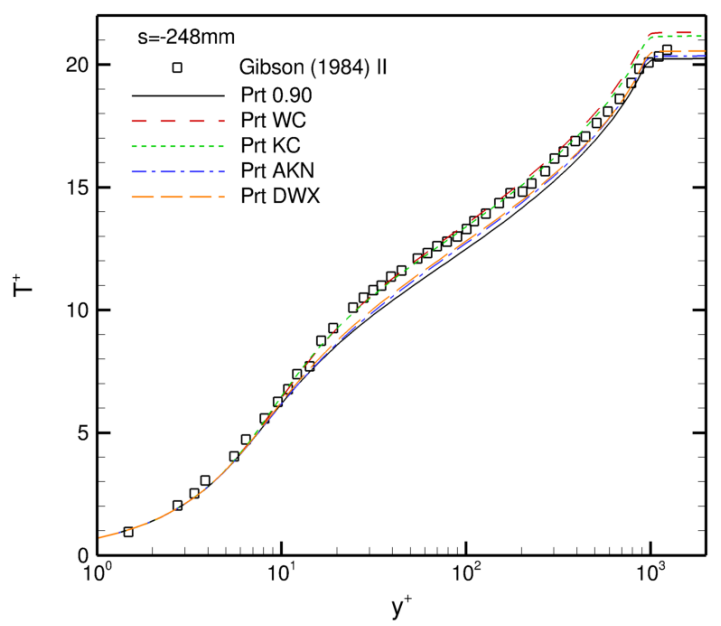

Figure 10: Temperature profile for Gibson boundary layer.

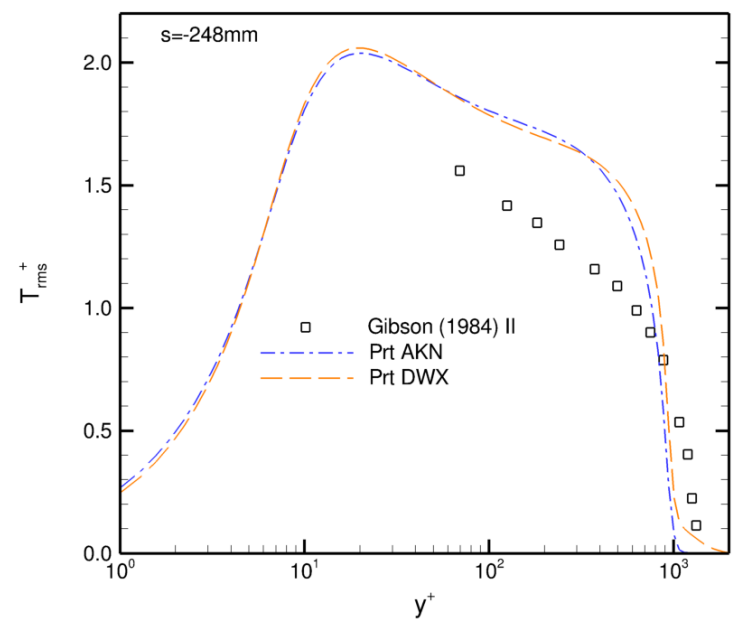

Figure 11: RMS temperature profile for Gibson boundary layer.

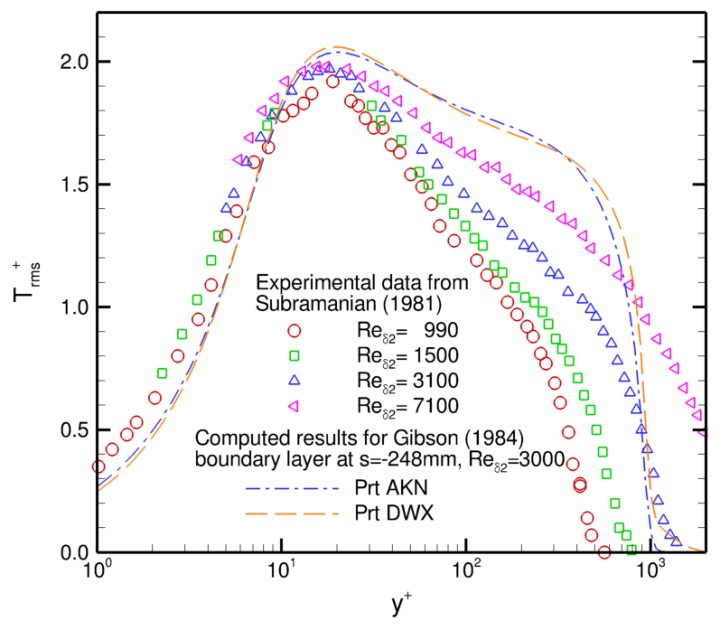

Figure 12: Comparison of RMS temperature with data of Subramanian(1981). 




Figure 13: Turbulent heat flux profile for Gibson boundary layer.

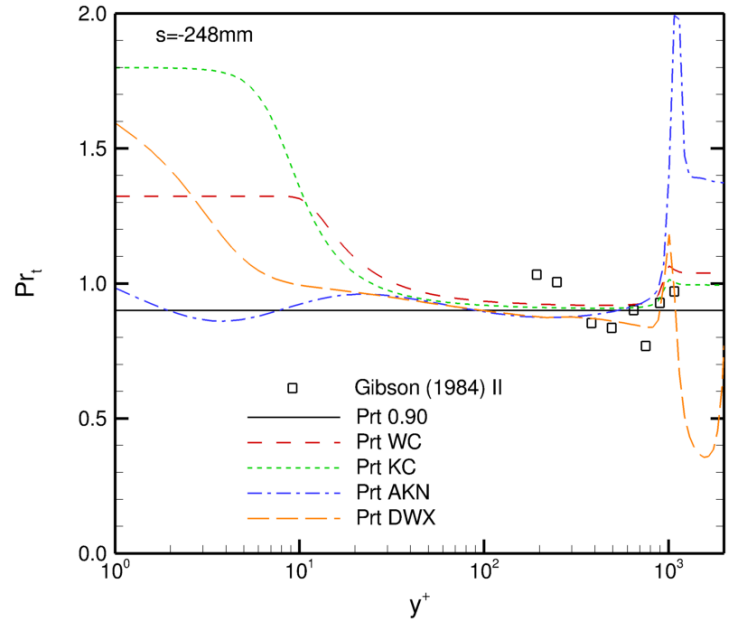

Figure 14: Turbulent Prandtl number profile for Gibson boundary layer.

\section{V.B. Pipe Thermal Entrance Region}

The experimental configuration of Hishida and Nagano ${ }^{41-43}$ consists of a long circular pipe with an upstream section that is thermally insulated and a downstream section with a $100^{\circ} \mathrm{C}$ isothermal wall. Thus the velocity field is already fully developed before the thermal boundary layer begins to form. In this case, Reynolds' analogy between turbulent heat and momentum transfer does not hold. The Reynolds number (based on bulk velocity and pipe diameter) is reported as 40000 , and at the entrance to the heated section $(x=0 \mathrm{~mm})$ the centerline velocity and temperature are nominally $17 \mathrm{~m} / \mathrm{s}$ and $26^{\circ} \mathrm{C}$.

Figure 15 presents the temperature profiles at three streamwise locations. The results generally follow the same trend observed in the boundary layer flows, with $\operatorname{Pr}_{T} 0.90$ providing the lowest values, the WC and $\mathrm{KC}$ zero-equation models providing the highest values, and the AKN and DWX two-equation models falling in the middle. However, for this case the AKN model displays some streamwise variation in the outer part of the boundary layer (i.e., the pipe centerline region). Overall, the results of the more sophisticated thermal variance models are only slightly better than those obtained using the constant turbulent Prandtl number. The WC and KC models continue to perform well, despite their relative simplicity.

Results for the RMS temperature profiles are shown in Figure 16. The temperature variance models reasonably predict the peak values and the profile shapes, with the DWX model being somewhat more accurate.

Profiles of turbulent heat flux, Figure 17, indicate good agreement in the outer part of the boundary layer. However, the values in the near-wall region appear to rise too rapidly as one moves away from the wall. Agreement with the peak values is generally good. The turbulent Prandtl number data shown in Figure 18 is similar to that of Blackwell. Although not shown, the log-layer data at the upstream locations is somewhat higher. This trend is not captured by the $\operatorname{Pr}_{T} 0.90$, WC, or $\mathrm{KC}$ models whose upstream profiles are essentially identical to that shown in Figure 18. Their agreement with the data at this location is therefore fortuitous. The AKN and DWX models do predict a streamwise variation of the log-layer $\operatorname{Pr}_{T}$. However, they continue to underpredict values within the sublayer.

\section{V.C. Axisymmetric Jets}

With the exception of References 21-23, thermal diffusivity models have not been widely applied to free shear flows. In this section, we therefore consider two subsonic heated axisymmetric jet cases. The first case is that of Lockwood-Moneib ${ }^{44}$ in which a fine-wire thermocouple was used to measure the mean and fluctuating temperature in a a jet of preheated air that emerged from a $19.3 \mathrm{~mm}$ diameter vertical pipe that was $1 \mathrm{~m}$ long. Fully developed pipe flow conditions existed at the exit of the pipe, with a jet exit velocity of $117 \mathrm{~m} / \mathrm{s}$ and Reynolds number based on pipe diameter of $5.0 \times 10^{4}$. Electrical trim heating was applied to 


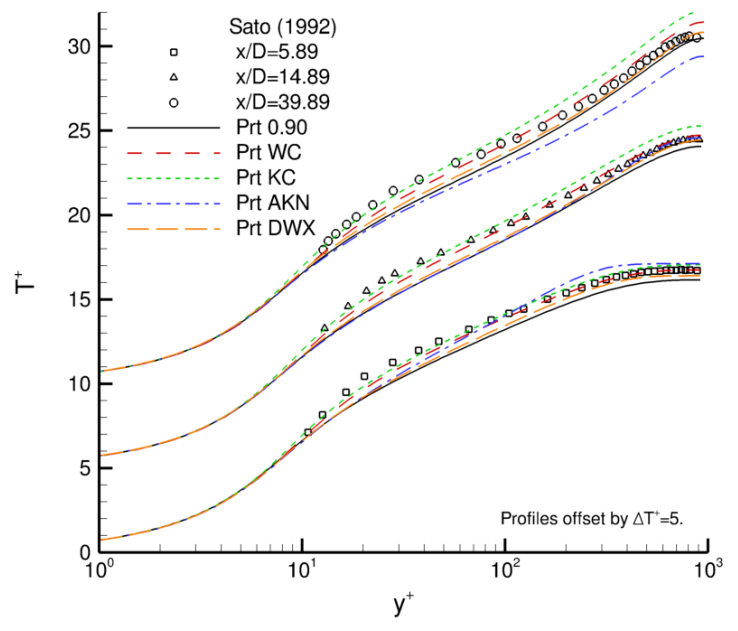

Figure 15: Temperature profiles for pipe flow.



Figure 16: RMS temperature profiles for pipe flow.

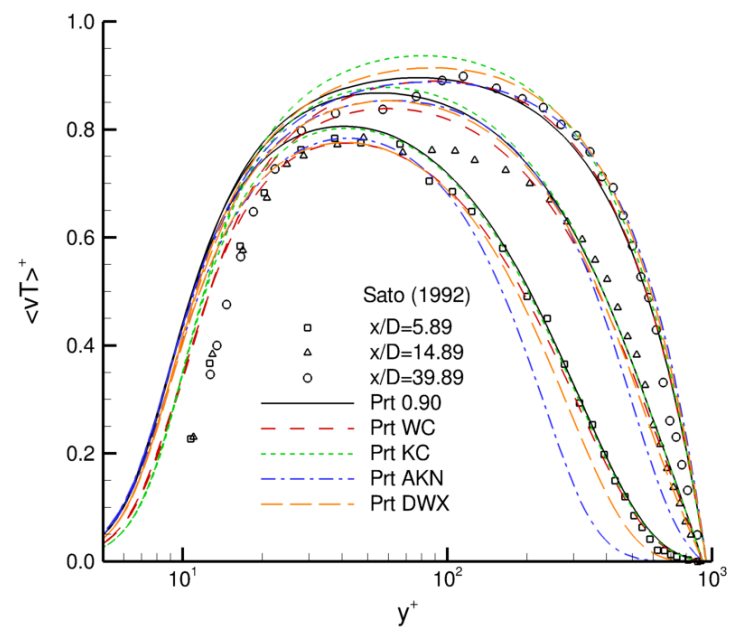

Figure 17: Heat flux profiles for pipe flow.

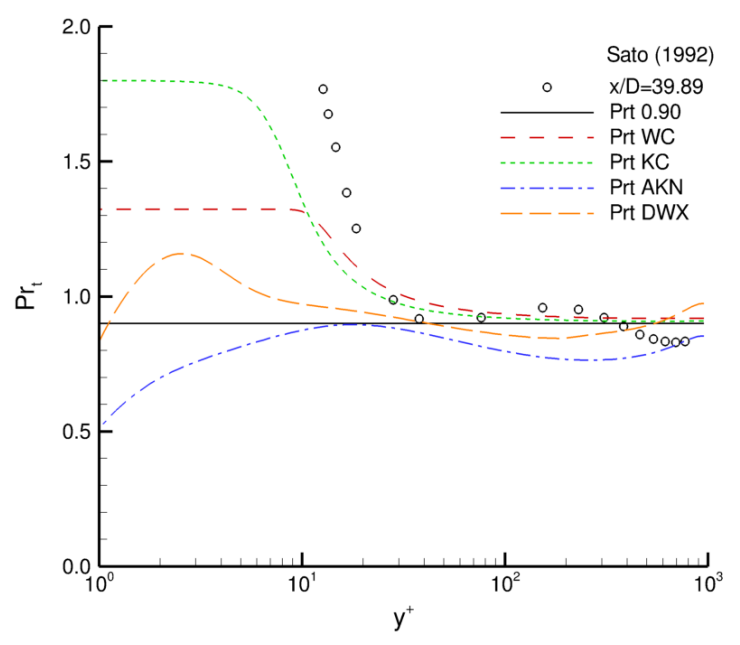

Figure 18: Turbulent Prandtl number profile for pipe flow. 
the surface of the pipe to maintain an essentially flat temperature profile. The jet temperature difference with the ambient air was $255 \mathrm{~K}$ yielding $T_{j} / T_{\infty}=1.86$, the jet Mach number was 0.25 , and the jet Reynolds number based on pipe diameter was 50000. For the jet calculations, a freestream turbulent Prandtl number of 0.70 was used. Results for the WC and $\mathrm{KC}$ models are not shown since, away from walls, these models essentially provide a constant $P r_{T}$.

Figure 19 compares the centerline temperature decay. Most of the models predict a thermal potential core that is approximately one diameter too long. The AKN model predicts the potential core length very well, but the downstream decay is much too rapid. The DWX results are found to be reasonably good, and the changes in the model coefficients made by BCD offer a significant improvement over the SSZ model. Growth of the half width of the thermal jet is shown in Figure 20. From this plot it is apparent that most of the models underpredict the width of the thermal jet. The AKN model is the lone exception, providing reasonably good agreement with the data.



Figure 19: Centerline temperature for Lockwood jet.

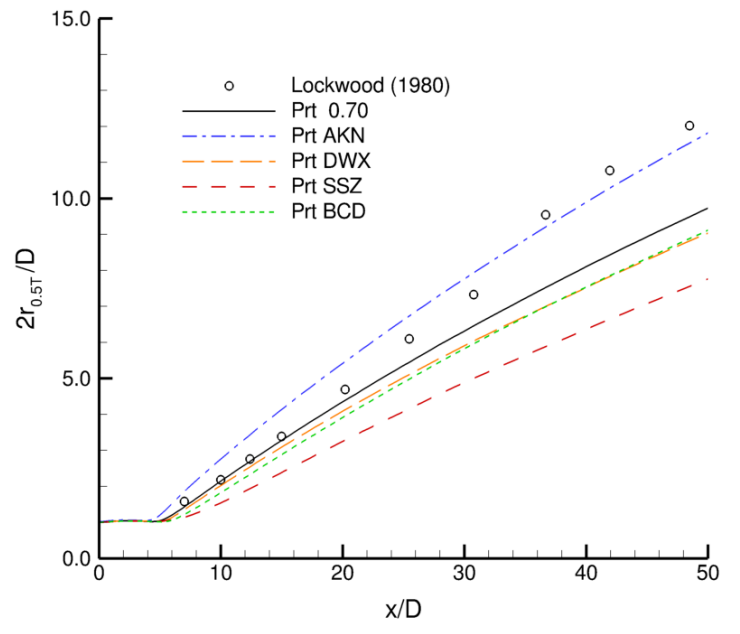

Figure 20: Axial growth of temperature profiles for Lockwood jet.

Centerline values of $T_{r m s}$ are plotted in Figures 21 and 22. The first plot is normalized with respect to the temperature difference at the jet exit plane, while the second is normalized by the local centerline temperature difference. Since the constant $\mathrm{Pr}_{T}$ model does not contain any turbulence closure expressions for $T_{r m s}$, that model is omitted from the plots. In both figures, the SSZ and BCD models provide the best agreement with the data. The AKN and DWX models that provided good agreement with the RMS temperature values in wall-bounded flow are found to be the worst models for this case. The AKN model, in particular, predicts values that are an order of magnitude too large. This disparity might be caused by the choice of mixed timescale. Of the models examined, the DWX model is the only one that uses a $\tau_{m}$ based on the harmonic average of the velocity and thermal timescales.

In Figures 23 and 24, we consider the mean and RMS temperature profiles at a downstream location of $x / D=41.93$. The profiles are normalized with respect to the thermal half width of the jet and the local centerline temperature difference. Within the context of these normalized profiles, all of the models provide good prediction of the mean temperature in the inner half of the jet. In the outer half, the DWX and BCD models are found to provide very good agreement, while the AKN and SSZ models under- and over-predict the temperature profile respectively. For the RMS temperature, the BCD model provides the best agreement with the data even though it underpredicts the peak $T_{r m s}$ value. The SSZ model is similar, but predicts higher values in the outer fraction of the jet. Notably higher values of $T_{r m s}$ are predicted by the DWX model, particularly near the centerline, and the profile for AKN appears completely inconsistent with that of the data or the other models.

The second jet case examined is the Acoustic Reference Nozzle (ARN), a convergent axisymmetric 2" diameter nozzle that has been tested ${ }^{45,46}$ over a range of heated and unheated operating conditions, Table 3. These cases have been identified for benchmarking turbulence model improvements because of the extensive mean and turbulent velocity statistics that have been measured with particle image velocimetry (PIV). The 


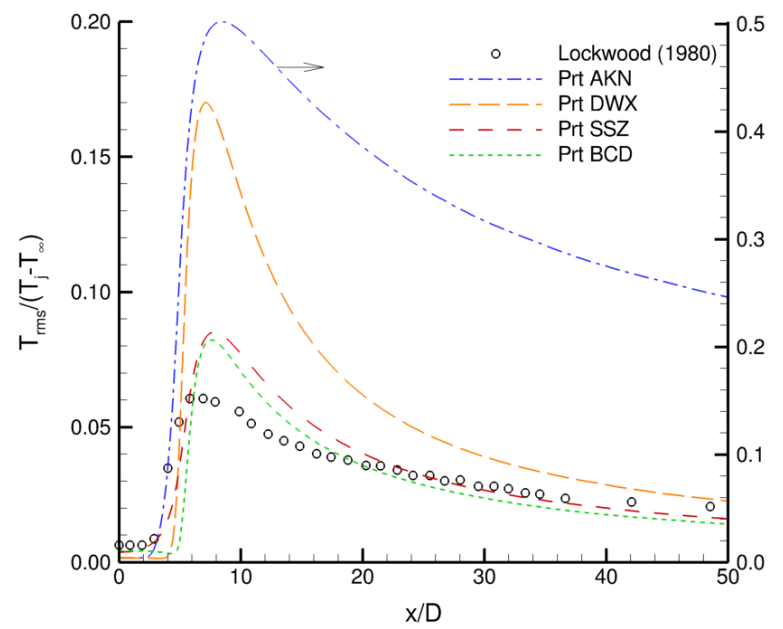

Figure 21: Centerline RMS temperature for Lockwood jet normalized by jet exit temperature difference.

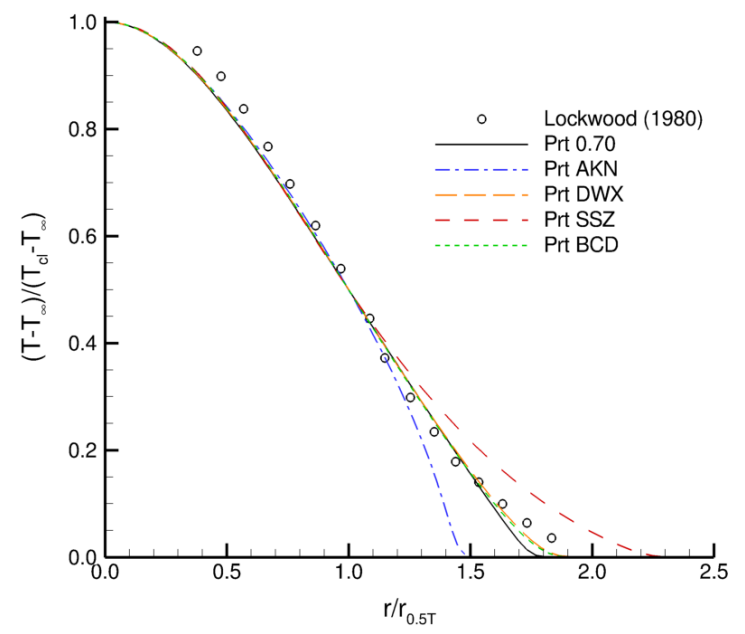

Figure 23: Temperature profile for Lockwood jet.

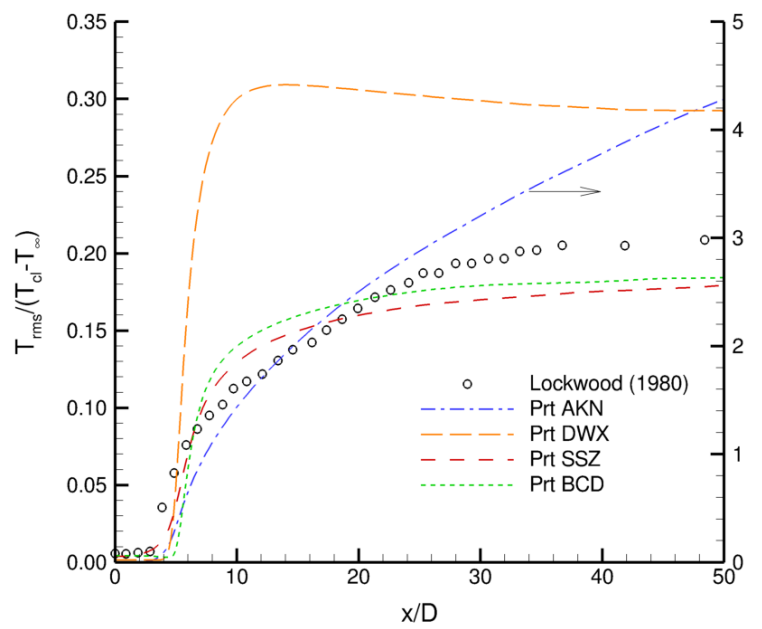

Figure 22: Centerline RMS temperature for Lockwood jet normalized by local centerline temperature difference.

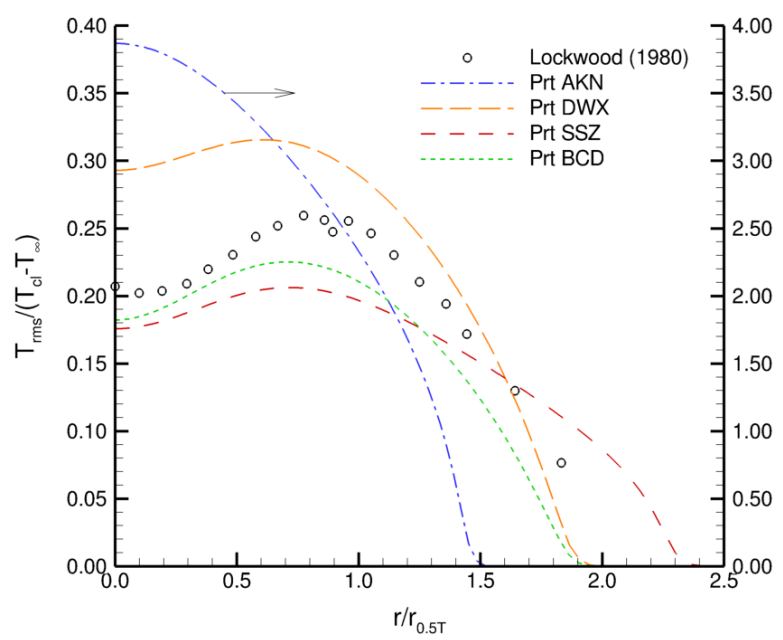

Figure 24: RMS temperature profile for Lockwood jet. 
experimental data for set points 3 and 23 indicate that heating the jet results in a shorter potential core of velocity. Results of Reynolds-averaged Navier-Stokes simulations ${ }^{47}$ indicate a shift in the right direction, though not as much as indicated by the data. It is thought that improvement in the thermal modeling of these jets might provide better prediction not only of the thermal flow field, but also of the velocity field.

Thermal data is not available for the ARN geometry. However, velocity and temperature data obtained by Rayleigh scattering are available in References 48 and 49 for a similar 2" diameter configuration referred to as the Small Metal Chevron (SMC) family of nozzles. The baseline nozzle from this series, SMC000, has a smooth round exit without chevrons. In Reference 46 it is shown that both nozzles produce similar velocity and turbulence statistics. Therefore, assuming the thermal statistics to be likewise similar, the Rayleigh scattering data for the SMC000 nozzle will be used to evaluate the current model predictions. The present results are for Setpoint 23, which has $T_{j} / T_{\infty}=1.760, M_{j}=0.37$ and $R e_{D}=2.0 \times 10^{5}$.

Table 3: Set point conditions for ARN axisymmetric jet.

\begin{tabular}{|clllll|}
\hline Set Point & NPR & $T_{t_{j}} / T_{\infty}$ & $T_{j} / T_{\infty}$ & $M_{a}$ & $M_{j}$ \\
\hline 3 & 1.197 & 1.000 & 0.950 & 0.50 & 0.51 \\
23 & 1.103 & 1.810 & 1.760 & 0.50 & 0.37 \\
42 & 1.066 & 2.750 & 2.700 & 0.50 & 0.30 \\
7 & 1.861 & 1.000 & 0.840 & 0.90 & 0.98 \\
27 & 1.361 & 1.922 & 1.760 & 0.90 & 0.68 \\
46 & 1.225 & 2.861 & 2.700 & 0.90 & 0.54 \\
29 & 1.900 & 2.114 & 1.760 & 1.33 & 1.00 \\
49 & 1.692 & 3.138 & 2.700 & 1.48 & 0.90 \\
\hline
\end{tabular}

Figures 25 and 26 compare the centerline velocity and temperature decay. The velocity decay is typical of previous studies that have shown $k-\epsilon$ models predicting a potential core that is too long. The temperature decay also indicates a predicted thermal potential core that is too long. Differences between the turbulent Prandtl number models are similar to those seen for the Lockwood jet, although there is a larger spread for this case.

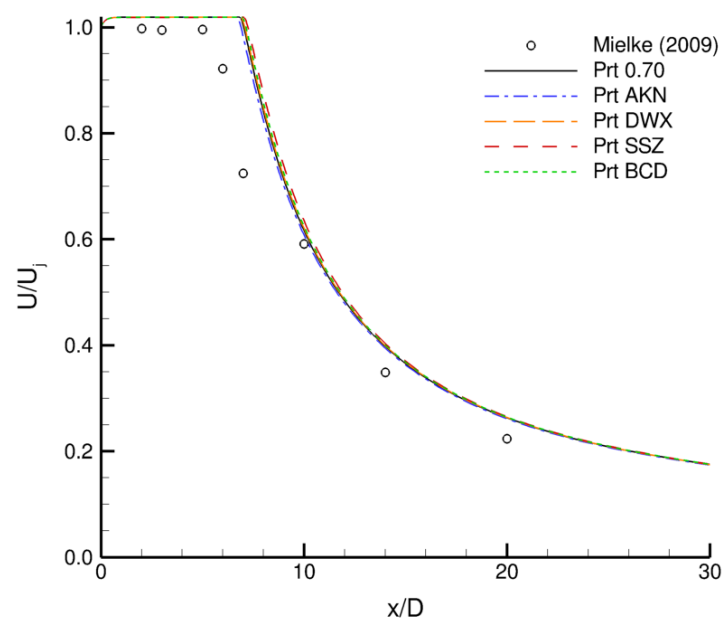

Figure 25: Centerline velocity for ARN jet.

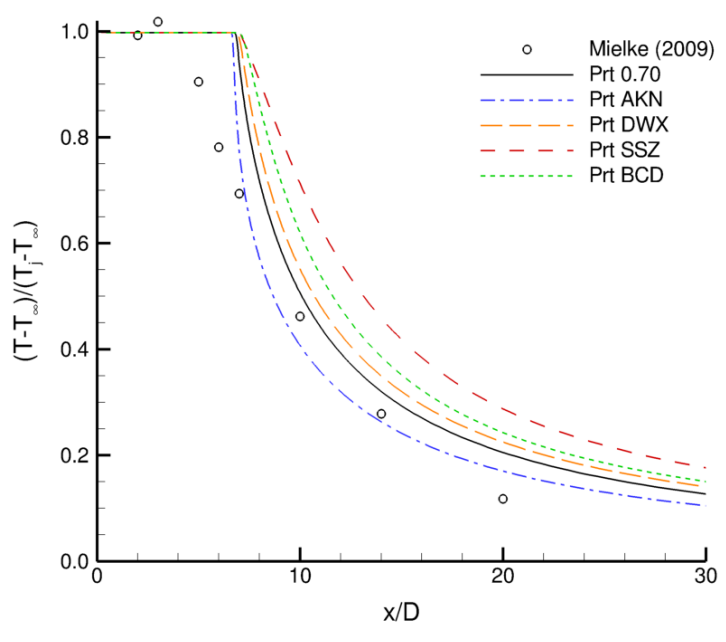

Figure 26: Centerline temperature for ARN jet.

Jet spreading rates of the velocity and thermal fields are plotted in Figures 27 and 28. Turbulent Prandtl number is found to have little effect on the velocity field. The trend in thermal growth rate between turbulent Prandtl models is consistent with the centerline decay, i.e., prediction of a slower growth rate leads to a longer and more gradual decay of the thermal potential core. The predicted thermal growth rates are very similar to those for the Lockwood jet, but the agreement with the data for this case is rather poor. Discussions with 
the experimentalists have revealed that the Rayleigh scattering data beyond $x / D>10$ may be affected by freestream particle contamination.

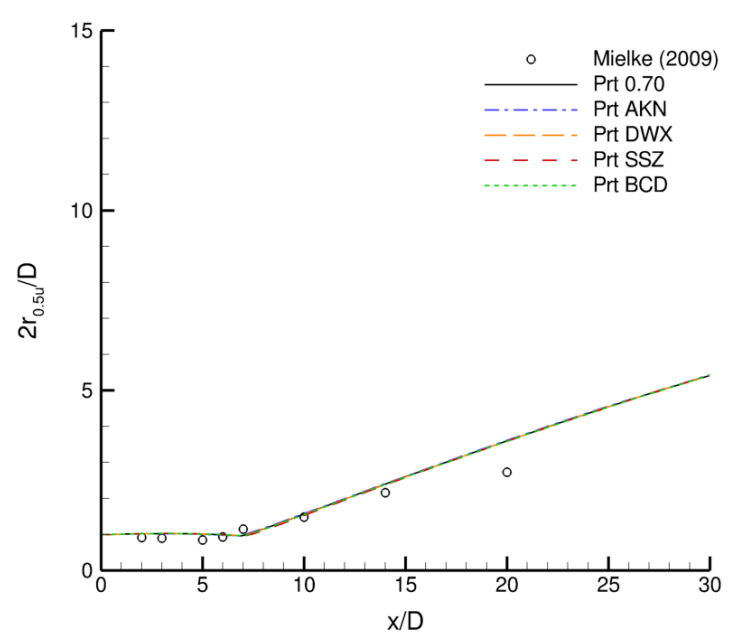

Figure 27: Axial growth of velocity profiles for ARN jet.

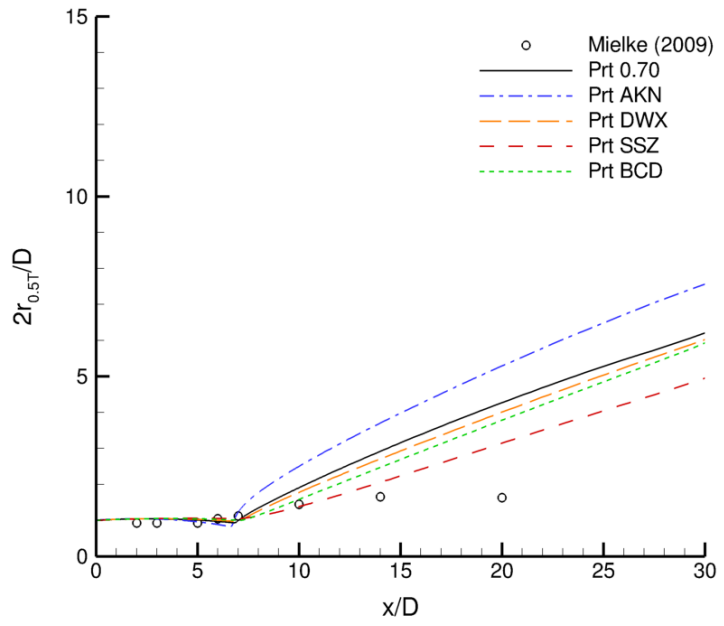

Figure 28: Axial growth of temperature profiles for ARN jet.

The centerline RMS temperature values shown in Figures 29 and 30 are also found to be in close agreement with the previous case. However, the data provided by the Rayleigh scattering technique is significantly higher than that measured by Lockwood. As suggested by the analytical work of George ${ }^{50}$ and later demonstrated in the experiments of References 51 and 52, differences in both the near-field and asymptotic values of a passive scalar like temperature are due to differences in the turbulent structure that begin at the jet exit. Smooth contraction nozzles tend to have more laminar-like exit profiles and exhibit more well-defined vortical structures in the plume that undergo the traditional roll-up, pairing, and breakdown process. This vortical action causes more rapid mixing of the mean temperature in both the near and far fields. The $a b$ solute intensity of the temperature fluctuations (i.e., normalized by the jet exit mean) indicates a centerline peak that is roughly double that found in a pipe-flow jet, while the centerline value of the relative temperature fluctuations (i.e., normalized by the local centerline mean) tends to asymptote roughly $8 \%$ higher. The Rayleigh data seems to support this trend, though the values are still substantially higher than observed in other experiments. The similarity in model results for the two cases suggests that the Reynolds-averaged technique may be incapable of representing such structure-driven flows.

For completeness, the normalized mean and RMS temperature profiles at the last measurement station of $x / D=20$ are shown in Figures 31 and 32. The mean temperature data in the outer half of the profile are lower than observed in the Lockwood case, while the relative $T_{r m s}$ data across the profile are substantially higher (by a factor of 6 or more). The predicted profile results are found to be similar to those for the Lockwood case.

For higher jet Mach numbers, where compressibility becomes a more significant factor, one would expect that changes in the thermal modeling would have a greater effect on the velocity in the plume. However, for these particular low subsonic jets, that is clearly not the case. This means that the differences observed in velocity data for low-speed unheated and heated jets cannot be explained within the framework of the present models. One way to achieve such a result might be to sensitize the $k-\epsilon$ turbulence model coefficients to either the timescale ratio $R$ or the turbulent Prandtl number. Additional insight from Direct Numerical Simulations or high resolution Large Eddy Simulations might be needed in order to provide a physical justification for such an extension of the models, particularly considering the excellent velocity profile agreement of current models in both the sublayer and log-layer regions where $\operatorname{Pr}_{T}$ has been shown to vary considerably. These results also indicate a significant shortcoming in modeling the difference in scalar fluctuations between pipeflow jets and those from smooth contraction nozzles. 


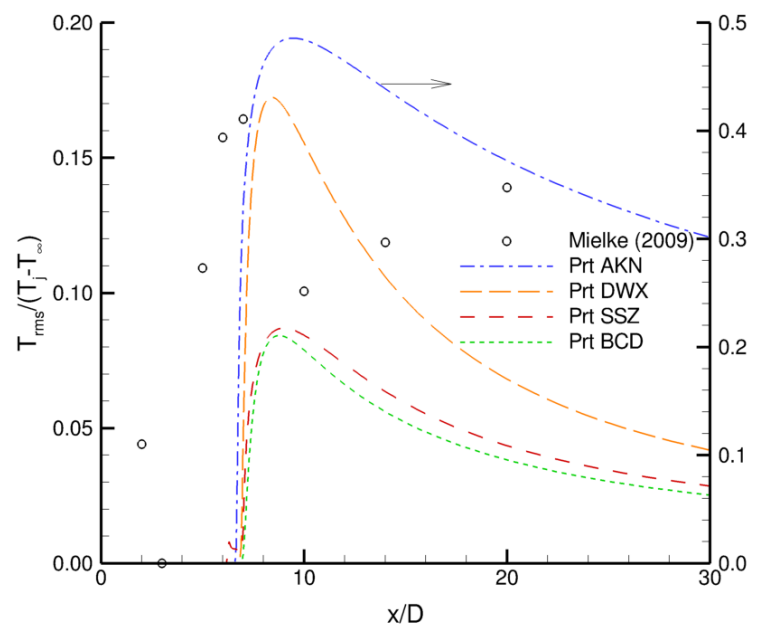

Figure 29: Centerline RMS temperature for ARN jet normalized by jet exit temperature difference.



Figure 30: Centerline RMS temperature for ARN jet normalized by local centerline temperature difference.

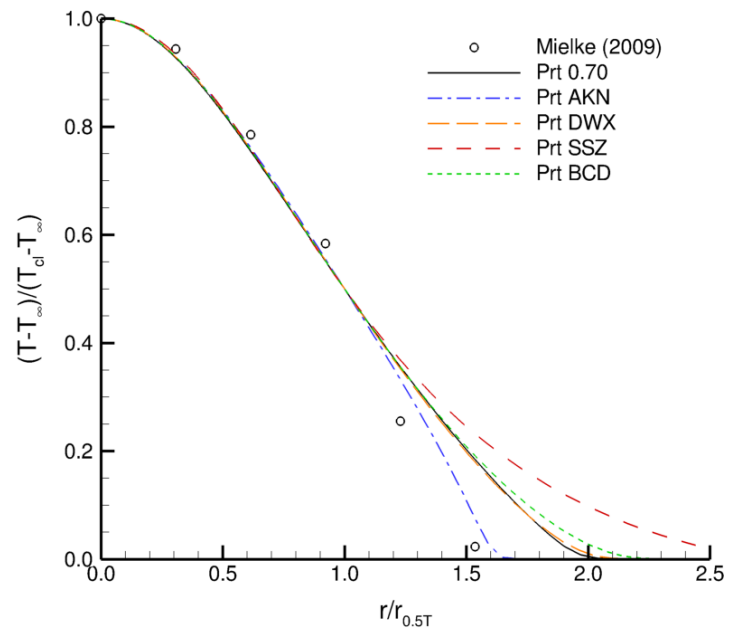

Figure 31: Temperature profile for ARN jet.

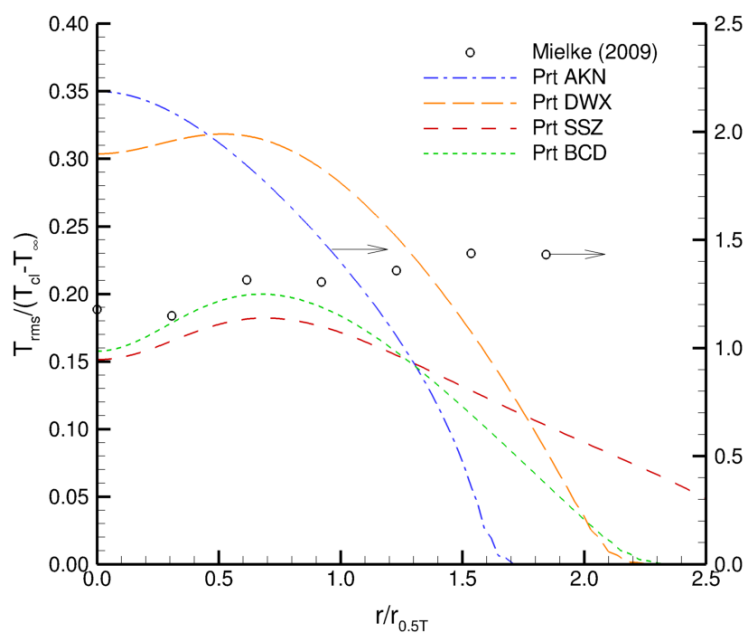

Figure 32: RMS temperature profile for ARN jet. 


\section{Conclusions}

In this study several variable turbulent Prandtl number formulations have been examined for three types of flow: boundary layer, pipe flow, and jets. The chosen models include simple algebraic formulations that relate the thermal diffusivity to the local turbulent viscosity as well as two-equation differential transport models for the thermal scalar variance and dissipation rate.

In the external boundary layer flows, the $\mathrm{WC}^{6}$ and $\mathrm{KC}^{5}$ zero-equation models are found to perform well, predicting an appropriate increase in turbulent Prandtl number very near the wall that appears to be a prerequisite for obtaining the proper offset in the temperature law of the wall. Results for the $\mathrm{AKN}^{16}$ and DWX $^{18}$ two-equation models showed good agreement in the prediction of the thermal variance in the boundary layer, but failed to reproduce the proper log-layer behavior of the mean temperature profiles. This likewise appears to be tied to an underprediction of the sublayer turbulent Prandtl number. Similar results were found for the thermal entrance region of a pipe. In this case, since the velocity field is fully developed before the thermal boundary layer begins to form, the turbulent Prandtl number should exhibit a streamwise variation. The $\mathrm{WC}$ and $\mathrm{KC}$ models fail to capture this trend, since their formulations are primarily based upon the turbulent viscosity. The AKN and DWX models do exhibit a streamwise variation in $P r_{T}$ in the log-layer region, but again underpredict values in the sublayer.

The effect of the WC and KC models appears to be limited to the near wall region, and for free shear flows the results are essentially the same as those obtained with a constant $P r_{T}$ approach. The AKN and DWX models were found to perform less successfully in the prediction of jet flows, yielding thermal variances higher than measured. The AKN model in particular deviated significantly from expectations, and one reason for this could be the choice of mixed timescale. The $\mathrm{SSZ}^{20}$ and $\mathrm{BCD}^{23}$ models were shown to be more accurate for jets, but numerical issues with the near-wall source terms precluded their evaluation for wall-bounded flow. Most of the models were found to underpredict the thermal growth rate of the jet. For the particular low subsonic heated jets examined, differences in the thermal modeling had no significant effect on the centerline velocity decay. The models were also found to predict very similar $T_{r m s}$ values in jet plumes downstream of straight pipes and smooth contraction nozzles. However, data indicate that the smooth contraction nozzles should produce both higher peak absolute intensities near the end of the thermal core region as well as higher relative intensities far downstream.

Overall, these results are less favorable than those found in previous publications. Part of the reason for this could be the use of uniform upstream conditions, from which the solutions are allowed to naturally develop, versus the use of prescribed inflow profiles from experimental data at a given measurement station. While the latter approach is quite useful for model validation, it does not provide an accurate assessment of the models for applications where such detailed information is not available. Further studies could be conducted to more closely examine the effect of inflow conditions. Following the practices outlined on the AIAA Turbulence Model Benchmarking website, ${ }^{53}$ these results should be verified through the use of another flow solver in order to identify any implementation-specific bias. In particular, these models seem to be sensitive to the treatment of the near-wall terms as evidenced by the difficulties encountered with the SSZ and BCD models. Furthermore, additional test cases should be considered. With the boundary layer and jet cases investigated herein, the momentum and thermal shear regions develop simultaneously. Other cases similar to the pipe thermal entrance problem that involve a change in the upstream or downstream thermal boundary conditions could better highlight the benefits of a variable turbulent Prandtl number formulation.

\section{References}

${ }^{1}$ Wilcox, D. C., Turbulence Modeling for CFD, DCW Industries, 2nd ed., 1998.

${ }^{2}$ Dembowski, M. A. and Georgiadis, N. J., "An Evaluation of Parameters Influencing Jet Mixing Using the WIND NavierStokes Code," NASA TM-211727, Aug. 2002.

${ }^{3}$ Georgiadis, N. J., Yoder, D. A., Vyas, M. A., and Engblom, W. A., "Status of Turbulence Modeling for Hypersonic Propulsion Flowpaths," NASA TM-2012-217277, April 2012.

${ }^{4}$ Kays, W. M., "Turbulent Prandtl Number - Where Are We?" Transactions of the ASME, Journal of Heat Transfer, Vol. 116, May 1994, pp. 284-295.

${ }^{5}$ Kays, W. M. and Crawford, M. E., Convective Heat and Mass Transfer, McGraw-Hill, New York, 3rd ed., 1993.

${ }^{6}$ Wassel, A. T. and Catton, I., "Calculation of Turbulent Boundary Layers Over Flat Plates with Different Phenomenological Theories of Turbulence and Variable Turbulent Prandtl Number," International Journal of Heat and Mass Transfer, Vol. 16, No. 8, 1973, pp. 1547-1563.

${ }^{7}$ Patel, V. C., Rodi, W., and Scheuerer, G., "Turbulence Models for Near-Wall and Low-Reynolds Number Flows: A Review," AIAA Journal, Vol. 23, No. 9, Sept. 1985, pp. 1308-1319. 
${ }^{8}$ Wilcox, D., "Reassessment of the Scale-Determining Equation for Advanced Turbulence Models," AIAA Journal, Vol. 26, No. 11, Nov. 1988, pp. 1299-1310.

${ }^{9}$ Lang, N. J. and Shih, T.-H., "Critical Comparison of Two-Equation Turbulence Models," NASA TM-105237, Sept. 1991, See also ICOMP-91-15, 1991; CMOTT-91-05, 1991.

${ }^{10}$ So, R., Lai, Y., and Zhang, H., "Near-Wall Variable-Prandlt-Number Turbulence Model for Compressible Flow," AIAA Journal, Vol. 29, No. 11, Nov. 1991, pp. 1819-1835.

${ }^{11}$ Speziale, C. G., "A Review of Reynolds Stress Models for Turbulent Shear Flows," NASA CR-195054, March 1995, See also ICASE Report No. 95-15.

${ }^{12}$ Thakur, S. and Shyy, W., "Reynolds Stress Models for Flows in Complex Geometries: Review and Application," AIAA Paper 99-3782, June 1999.

${ }^{13}$ Nagano, Y. and Kim, C., "A Two-Equation Model for Heat Transport in Wall Turbulent Shear Flows," Journal of Heat Transfer, Vol. 110, Aug. 1988, pp. 583-589.

${ }^{14}$ Nagano, Y., Tagawa, M., and Tsuji, T., "An Improved Two-Equation Heat Transfer Model for Wall Turbulent Shear Flows," ASME/JSME Thermal Engineering Proceedings, Vol. 3, 1991, pp. 233-240.

${ }^{15}$ Youssef, M., Nagano, Y., and Tagawa, M., "A Two-Equation Heat Transfer Model for Predicting Turbulent Thermal Fields Under Arbitrary Wall Thermal Conditions," International Journal of Heat and Mass Transfer, Vol. 35, No. 11, 1992, pp. 3095-3104.

${ }^{16}$ Abe, K., Kondoh, T., and Nagano, Y., "A New Turbulence Model for predicting Fluid Flow and Heat Transfer in Separating and Reattaching Flows-II. Thermal Field Calculations," International Journal of Heat and Mass Transfer, Vol. 38, No. 8, 1995, pp. 1467-1481.

${ }^{17}$ Nagano, Y., Hattori, H., and Abe, K., "Modeling the Turbulent Heat and Momentum Transfer in Flows Under Different Thermal Conditions," Fluid Dynamics Research, Vol. 20, 1997, pp. 127-142.

${ }^{18}$ Deng, B., Wu, W., and Xi, S., "A Near-Wall Two-Equation Heat Transfer Model for Wall Turbulent Flows," International Journal of Heat and Mass Transfer, Vol. 44, No. 4, 2000, pp. 691-698.

${ }^{19}$ Sommer, T., So, R., and Lai, Y., "A Near-Wall Two-Equation Model for Turbulent Heat Fluxes," International Journal of Heat and Mass Transfer, Vol. 35, No. 12, 1992, pp. 3375-3387.

${ }^{20}$ Sommer, T., So, R., and Zhang, H., "Near-Wall Variable-Prandlt-Number Turbulence Model for Compressible Flow," AIA A Journal, Vol. 31, No. 1, Jan. 1993, pp. 27-35.

${ }^{21}$ Chidambaram, N., Dash, S. M., and Kenzakowski, D. C., "Scalar Variance Transport in the Turbulence Modeling of Propulsive Jets," Journal of Propulsion and Power, Vol. 17, No. 1, 2001, pp. 79-84.

${ }^{22}$ Kenzakowski, D., "RANS Modeling Improvements for Jets Using Scalar Variance Equations," AIAA Paper 2006-0491, Jan. 2006.

${ }^{23}$ Brinckman, K. W., Calhoon, W. H. J., and Dash, S. M., "Scalar Fluctuation Modeling for High-Speed Aeropropulsive Flows," AIAA Journal, Vol. 45, No. 5, May 2007, pp. 1036-1046.

${ }^{24}$ So, R. and Sommer, T., "A Near-Wall Eddy Conductivity Model for Fluids with Different Prandtl Numbers," Journal of Heat Transfer, Vol. 116, Nov. 1994, pp. 844-854.

${ }^{25}$ Sommer, T., So, R., and Zhang, H., "Supersonic Flow Calculations Using a Reynolds-Stress and a Thermal Eddy Diffusivity Turbulence Model," Journal of Fluids Engineering, Vol. 116, Sept. 1994, pp. 469-476.

${ }^{26}$ Sommer, T., So, R., and Zhang, H., "Heat Transfer Modeling and the Assumption of Zero Wall Temperature Fluctuations," Transactions of the ASME, Journal of Heat Transfer, Vol. 116, Nov. 1994, pp. 855-863.

${ }^{27}$ Cebeci, T. and Bradshaw, P., Physical and Computational Aspects of Convective Heat Transfer, Springer-Verlag, 1988.

${ }^{28}$ Anderson, J. D., Hypersonic and High Temperature Gas Dynamics, McGraw-Hill, Inc., New York, 1989.

${ }^{29}$ Xiao, X., Edwards, J. R., Hassan, H. A., and Gaffney, Jr., R. L., "Role of Turbulent Prandtl Number on Heat Flux at Hypersonic Mach Numbers," AIAA Paper 2005-1098, Jan. 2005.

${ }^{30}$ Robinson, D. and Hassan, H., "Further Development of the $k-\zeta$ (Enstrophy) Turbulence Closure Model," AIAA Journal, Vol. 36, No. 10, Oct. 1998, pp. 1825-1833.

${ }^{31}$ Xiao, X., Hassan, H. A., and Baurle, R. A., "Modeling Scramjet Flows with Variable Turbulent Prandtl and Schmidt Numbers," AIAA Journal, Vol. 45, No. 6, June 2007, pp. 1415-1423, See also AIAA Paper 2006-0128.

${ }^{32}$ Nelson, C., "An Overview of the NPARC Alliance's Wind-US Flow Solver," AIAA Paper 2010-0027, Jan. 2010.

${ }^{33}$ Yoder, D. A. and Georgiadis, N. J., "Implementation and Validation of the Chien $k-\epsilon$ Turbulence Model in the WIND Navier-Stokes Code," NASA TM-1999-209080, April 1999, See also AIAA Paper 99-0745.

${ }^{34}$ Abe, K., Kondoh, T., and Nagano, Y., "A New Turbulence Model for predicting Fluid Flow and Heat Transfer in Separating and Reattaching Flows-I. Flow Field Calculations," International Journal of Heat and Mass Transfer, Vol. 37, No. 1, 1994, pp. 139-151.

${ }^{35}$ Brinckman, K. W., Kenzakowski, D. C., and Dash, S. M., "Progress in Practical Scalar Fluctuation Modeling for HighSpeed Aeropropulsive Flows," AIAA Paper 2005-0508, Jan. 2005.

${ }^{36}$ Blackwell, B., Kays, W., and Moffat, R., "The Turbulent Boundary Layer on a Porous Plate: An Experimental Study of the Heat Transfer Behavior with Adverse Pressure Gradients," NASA CR-130291, Aug. 1972, Also labeled as Report HMT-16, Thermosciences Division, Department of Mechanical Engineering, Stanford University.

${ }^{37}$ Gibson, M., Verriopoulos, C., and Vlachos, N., "Turbulent Boundary Layer on a Mildly Curved Convex Surface. Part 1: Mean Flow and Turbulence Measurements," Experiments in Fluids, Vol. 2, No. 1, 1984, pp. 17-24.

${ }^{38}$ Gibson, M. and Verriopoulos, C., "Turbulent Boundary Layer on a Mildly Curved Convex Surface. Part 2: Temperature Field Measurements," Experiments in Fluids, Vol. 2, No. 2, 1984, pp. 73-80.

${ }^{39}$ Gibson, M., Verriopoulos, C., and Nagano, Y., "Measurements in the Heated Turbulent Boundary Layer on a Mildly Curved Convex Surface," Turbulent Shear Flows 3, edited by L. J. Bradbury, F. Durst, B. E. Launder, F. W. Schmidt, and J. H. Whitelaw, Springer Berlin Heidelberg, 1982, pp. 80-89. 
${ }^{40}$ Subramanian, C. S. and Antonia, A., "Effect of Reynolds Number on a Slightly Heated Turbulent Boundary Layer," International Journal of Heat and Mass Transfer, Vol. 24, 1981, pp. 1833-1846.

${ }^{41}$ Hishida, M. and Nagano, Y., "Structure of Turbulent Temperature and Velocity Fluctuations in the Thermal Entrance Region of a Pipe," Proceedins of the 6th International Heat Transfer Converence, Vol. 2, Hemishpere, Washington, DC, Aug. 1978, pp. 531-536.

${ }^{42}$ Sato, H., Nagano, Y., and Tagawa, M., "Distributions of Turbulence Quantities and Their Production, Diffusion, and Dissipation in the Thermal Entrance Region of a Pipe," Transactions of the Japan Society of Mechanical Engineers, Series B, Vol. 58, No. 553, 1992, pp. 2840-2847, In Japanese.

${ }^{43}$ Nagano, Y., Sato, H., and Tagawa, M., "Structure of Heat Transfer in the Thermal Layer Growing in a Fully Developed Turbulent Flow," Turbulent Shear Flows 9, edited by F. Durst, N. Kasagi, B. E. Launder, F. W. Schmidt, K. Suzuki, and J. H. Whitelaw, Springer-Verlag, Berlin, 1995, pp. 343-363, Selected Papers from the Ninth International Symposium on Turbulent Shear Flows, Kyoto, Japan, Aug 16-18, 1993.

${ }^{44}$ Lockwood, F. C. and Moneib, H. A., "Fluctuating Temperature Measurements in a Heated Round Free Jet," Combustion Science and Technology, Vol. 22, 1980, pp. 63-81.

${ }^{45}$ Bridges, J. E. and Wernet, M. P., "Measurements of the Aeroacoustic Sound Source in Hot Jets," AIAA Paper 2003-3130, May 2003.

${ }^{46}$ Bridges, J. E. and Wernet, M. P., "The NASA Subsonic Jet Particle Image Velocimetry (PIV) Dataset," NASA TM2011-216807, Nov. 2011.

${ }^{47}$ Georgiadis, N. J. and DeBonis, J. R., "Navier-Stokes Analysis Methods for Turbulent Jet Flows with Application to Aircraft Exhaust Nozzles," Progress in Aerospace Sciences, Vol. 42, 2006, pp. 377-418.

${ }^{48}$ Mielke, A. F., Elam, K. A., and Sung, C.-J., "MultiProperty Measurements at High Sampling Rates Using Rayleigh Scattering," AIAA Journal, Vol. 47, No. 4, April 2009, pp. 850-862, Presented as AIAA Paper 2008-0262.

${ }^{49}$ Mielke, A. F. and Elam, K. A., "Dynamic measurement of temperature, velocity, and density in hot jets using Rayleigh scattering," Experiments in Fluids, Vol. 47, No. 4-5, 2009, pp. 673-688, Presented at the 14th International Symposium on Applications of Laser Techniques to Fluid Mechanics, Lisbon, Portugal, 710 July 2008.

${ }^{50}$ George, W. K., "The Self-Similarity of Turbulent Flows and Its Relation to Initial Conditions and Coherent Structures," Advances in Turbulence, edited by W. K. George and R. E. A. Arndt, Hemisphere Publishing Corporation., 1989, pp. 39-73.

${ }^{51} \mathrm{Mi}$, J., Nobes, D. S., and Nathan, G. J., "Influence of Jet Exit Conditions on the Passive Scalar Field of an Axisymmetric Free Jet," Journal of Fluid Mechanics, Vol. 432, 2001, pp. 91-125.

${ }^{52} \mathrm{Xu}, \mathrm{G}$. and Antonia, R., "Effect of Initial Conditions on the Temperature Field of a Turbulent Round Free Jet," International Communications in Heat and Mass Transfer, Vol. 29, No. 8, 2002, pp. 1057-1068.

${ }^{53}$ Rumsey, C. L., "AIAA Tubulence Model Benchmarking Website," http://turbmodels.larc.nasa.gov, Accessed: 201511-10. 